NISTIR 8198r1

\title{
Improving Automated Insertion Applications by Restoring Rigid-Body Condition in Point-Based Registration
}

\author{
Geraldine S. Cheok \\ Marek Franaszek \\ Karl Van Wyk \\ Jeremy A. Marvel
}




\title{
Improving Automated Insertion Applications by Restoring Rigid-Body Condition in Point-Based Registration
}

\author{
Geraldine S. Cheok \\ Marek Franaszek \\ Karl Van Wyk \\ Jeremy A. Marvel \\ Intelligent Systems Division \\ Engineering Laboratory
}

This publication is available free of charge from:

https://doi.org/10.6028/NIST.IR.8198r1

March 2018

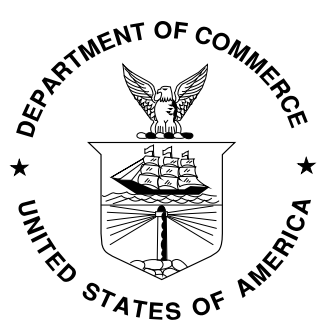

U.S. Department of Commerce Wilbur L. Ross, Jr., Secretary

National Institute of Standards and Technology Walter Copan, NIST Director and Under Secretary of Commerce for Standards and Technology 


\section{Contents}

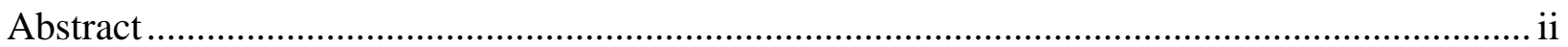

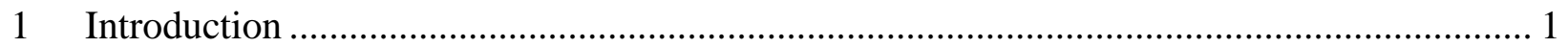

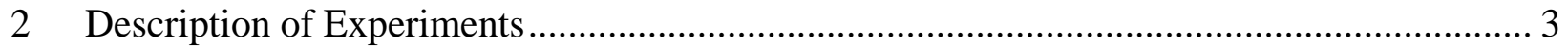

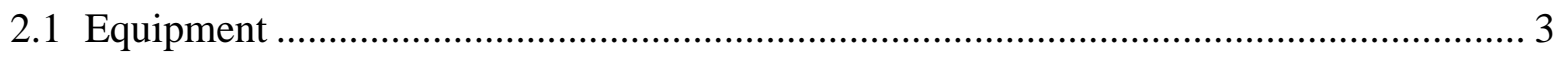

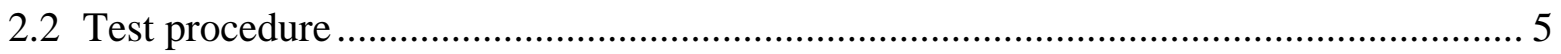

2.2.1 General procedure and concept of experiments ............................................ 5

2.2.2 Experiment 1, Runs 1-7 ........................................................................... 7

2.2.3 Experiment 2, Runs 9-15 ........................................................................... 7

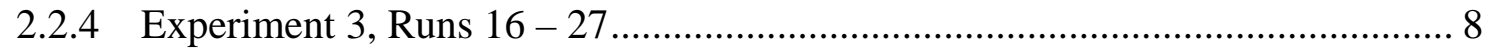

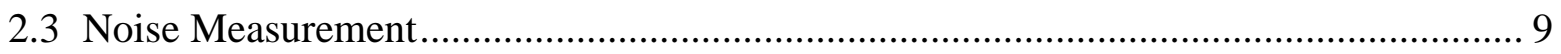

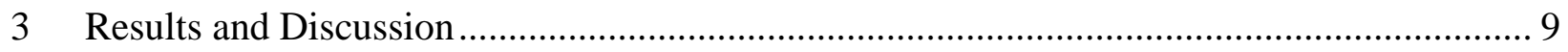

3.1 Failure Rate: Conventional Registration vs. RRBC Mehod ...................................... 9

3.2 Local Registration Method ..................................................................................... 14

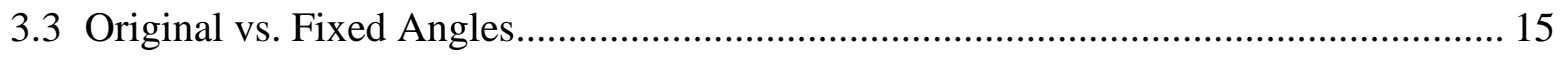

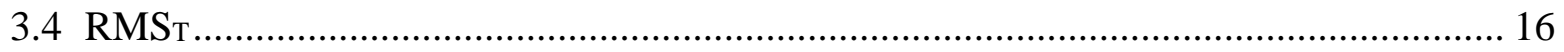

3.5 Target Registration Error (TRE) …................................................................ 16

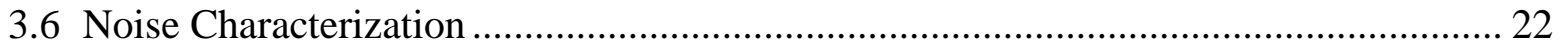

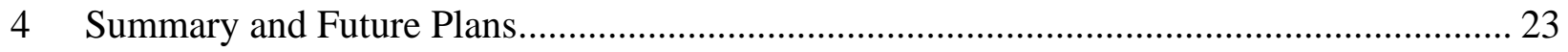

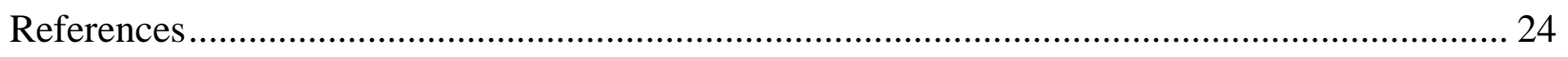

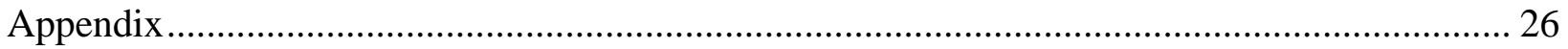




\begin{abstract}
Accurate knowledge of an object's location is essential in automated robotic applications such as machine tending, pick-and-place operations, and part assembly. Perception systems are used to acquire the object's location, and this information is then passed on to the robot. However, the measured location of the object is in the coordinate frame of the perception system. For the robot to act on the object, the location of the object must be transformed relative to the coordinate frame of the robot. In manufacturing applications that involve industrial robots, a common way to register coordinate frames to each other or to a global frame is by rigid-body, point-based registration using at least three common points or fiducials that are measured in both frames. Researchers at the National Institute of Standards and Technology (NIST) have developed a method to improve this registration method. The new method restores the rigidbody condition (RRBC).

This report presents an assessment of the NIST RRBC method by conducting practical robotic peg-in-hole experiments. In these experiments, a robot arm was used to insert a peg into a hole. A simple Pass/Fail metric was used to evaluate the effectiveness of the method. Two other metrics involving registration errors were also used. The results show that the RRBC reduces the number of failed insertions, especially with tight tolerances.
\end{abstract}

Keywords: peg-in-hole experiments; registration; registration error; rigid-body; target registration error. 


\section{INTRODUCTION}

Accurate knowledge of an object's location is essential in manufacturing-based robotic applications such as machine tending, pick-and-place operations, and part assembly. Perception systems are used to acquire the object's location, and this information is then passed on to the robot. However, the measured location of the object is in the coordinate frame of the perception system. For the robot to act on the object, the location of the object with respect to the perception coordinate frame must be transformed to the coordinate frame of the robot. This can be performed using a direct transformation or an intermediate transformation to a global coordinate frame where the required transformation matrix $\{\boldsymbol{R}, \boldsymbol{\tau}\}$ consists of a $3 \times 3$ rotation matrix, $\boldsymbol{R}$, and a 3x1 matrix, $\boldsymbol{\tau}$, is determined by a registration process.

In manufacturing applications that involve industrial robots, a common way to register coordinate frames to each other or to a global frame is by rigid-body, point-based registration using at least three common points or fiducials that are measured in both frames. In this report, the first coordinate frame, from which points are transformed, is called the working frame. The second frame, to which points are transformed, is called the destination frame. The registration process minimizes an error function, FRE (Fiducial Registration Error), so that the distances between corresponding $N$ fiducials measured in the destination frame, $\{\boldsymbol{Y}\}_{J}$, and the fiducials in the working frame, $\{\boldsymbol{X}\}_{J}$, transformed by $\{\boldsymbol{R}, \boldsymbol{\tau}\}$ to the destination frame are minimized as follows:

$$
F R E(\boldsymbol{R}, \boldsymbol{\tau})=\sqrt{\frac{1}{J} \sum_{j=1}^{J}\left\|\boldsymbol{R} \boldsymbol{X}_{j}+\boldsymbol{\tau}-\boldsymbol{Y}_{j}\right\|^{2}}
$$

Once the registration is completed and the transformation $\{\boldsymbol{R}, \boldsymbol{\tau}\}$ determined, the transformation can be used to transform other points $\left\{\boldsymbol{T}_{X}\right\}$ from the working frame to the destination frame. These points, called target points, are not used for registration and, in principle, they are measured only in the working frame. Example applications that leverage target points include peg-in-hole insertions [1], robotic welding and Cartesian path planning [2], obtaining hand-eye coordination [3], and grasp planning [4].

Increased position accuracy is sometimes needed to support precision robot applications such as component assembly. Sources of errors in position can be attributed to the perception system, robot kinematics, and the registration process. Registration error may be reduced by using an instrument that is accurate with small measurement uncertainty to reduce the error and noise of the measured fiducials. Additionally, registration error may be reduced by using a calibrated instrument to eliminate systematic errors and by proper selection of fiducials [5].

Another method of improving point-based registration requiring the measurement of many (> 100) fiducials was proposed in [6]. This method restores the rigid-body condition (RRBC) by applying corrections to the locations of fiducials in the working frame only. The corrections are 
calculated based on measurements of the fiducials in the working and destination frames. The aim of the method is to restore the rigid-body condition and assure that fiducials in the working frame can be mapped exactly onto the corresponding fiducials in the destination frame. Although the bias may only be present in the destination frame, the method works as though the bias was exclusively in the working frame and treats the mean locations of the fiducials in the destination frame as "ground truth". The method compensates for such bias in the fiducials $\{\boldsymbol{X}\}_{J}$ in the working frame. Then, the resulting residual value of the error function $E$ is reduced to zero (within numerical round-off error). Once the correction $\boldsymbol{\varepsilon}_{j}$ at each fiducial $\boldsymbol{X}_{j}$ is evaluated, the correction to any target location $\boldsymbol{T}_{X}$ is approximated by selecting four fiducials $\left\{\boldsymbol{X}_{j 1}, \boldsymbol{X}_{j 2}, \boldsymbol{X}_{j 3}, \boldsymbol{X}_{j 4}\right\}$ that are not co-linear, which form the smallest tetrahedron containing $\boldsymbol{T}_{X}$, and then linearly interpolating the four corrections $\left\{\boldsymbol{\varepsilon}_{j 1}, \boldsymbol{\varepsilon}_{j 2}, \boldsymbol{\varepsilon}_{j 3}, \boldsymbol{\varepsilon}_{j 4}\right\}$. This allows for the correction of the target location in the working frame and the reduction of the Target Registration Error, TRE ${ }^{1}$ [7]. Once corrected, the target points can be transformed to the destination frame using any three of the corrected fiducials to obtain $\{\boldsymbol{R}, \boldsymbol{\tau}\}$.

An extensive set of experimental data obtained from three different instruments was used to verify the method described above [6]. The data consisted of 125 fiducials (5 x 5 x 5 grid volume) and 16 target points in a work volume that was approximately ( $3 \times 3 \times 1.8$ ) $\mathrm{m}$. It was shown that the method reduced the $R M S_{T}$, Root Mean Square of $T R E(k)$, by about $75 \%$ for an instrument with a large bias and low noise and by about $30 \%$ for an instrument with low bias and large noise. $R M S_{T}$ is defined as:

$$
R M S_{T}=\sqrt{\frac{1}{K} \sum_{k=1}^{K} T R E^{2}(k)}
$$

where $K$ is the number of targets. TRE is defined as

$$
\operatorname{TRE}\left(\boldsymbol{T}_{\boldsymbol{x}}\right)=\left\|\boldsymbol{R} \boldsymbol{T}_{\boldsymbol{x}}+\boldsymbol{\tau}-\boldsymbol{T}_{\boldsymbol{y}}\right\|, \quad \text { Eq. } 3
$$

where $\boldsymbol{T}_{\boldsymbol{x}}$ and $\boldsymbol{T}_{\boldsymbol{y}}$ are the target positions in the working and destination frames, respectively.

To assess the RRBC method in a practical robotic application, three peg-in-hole experiments were conducted. In these experiments, a robot arm was used to insert a peg into a hole where the coordinates of the hole were obtained by registering a world coordinate frame to the robot coordinate frame. The experiments were conducted using a registration calculated using Eq. 1 and a registration calculated using the RRBC method. A simple Pass/Fail metric was used to

\footnotetext{
${ }^{1} T R E$ is the preferred metric for quantifying the quality of registration as opposed to FRE since TRE is the error at target points which are points of interest to practitioners. However, TRE requires measurement of the targets in the destination frame which may not be possible.
} 
evaluate the effectiveness of the method at each peg insertion location. In addition, two other metrics, $R M S_{T}$ and TRE, were also used.

This report presents the results from the three peg-in-hole experiments. The experimental set-up and test procedures are described in Section 2. The results and a discussion of the results are presented in Section 3. A summary, conclusions, and future plans are presented in Section 4.

\section{DESCRIPTION OF EXPERIMENTS}

A six degree of freedom (6 DOF), collaborative robot arm operating under position control was used to insert a peg into a series of holes. The robot arm used was very stiff. The coordinates of the holes were determined using three methods: conventional registration, RRBC, and a local registration method (see Section 2.2.3). A simple Pass/Fail metric was used to determine the effectiveness of a registration method.

Three experiments, Experiments 1 to 3, were conducted; each with slight variations. Each experiment was repeated at least seven times. The following sections describe the equipment used and each of the experiments in more detail.

\subsection{EQUIPMENT}

In the experiments, a $12.675 \mathrm{~mm}$ (0.499 in) diameter peg made of A2 tool steel was used and was rigidly attached to the robot arm (see Figure 1c). For Experiment 1, the end of the peg was conical, and for Experiments 2 and 3, the end of the peg was flat with a slight chamfer (see Figure 1a and b). From observations in Experiment 1, the peg was successfully inserted even though there was a very visible misalignment of the peg and the hole; however, the "chamfer" of the conical peg allowed the peg to be inserted without exceeding the preset force threshold. After Experiment 1, it was decided that a flat peg may make the distinction between Pass and Fail more definitive. Another reason for the change was that a flat peg would be more typical in practice.

The nominal tolerance on the diameter of the hole was $0.051 \mathrm{~mm}$ (0.002 in). The hole was drilled into a $28.575 \mathrm{~mm}$ (1 1/8 in) diameter cylindrical piece of aluminum (Figure 1c) and will be called an adapter, henceforth. 


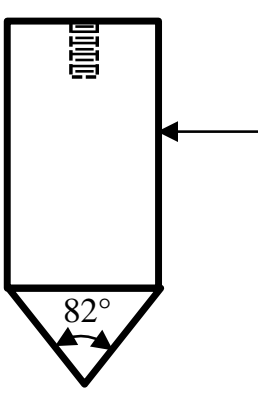

a. Conical peg

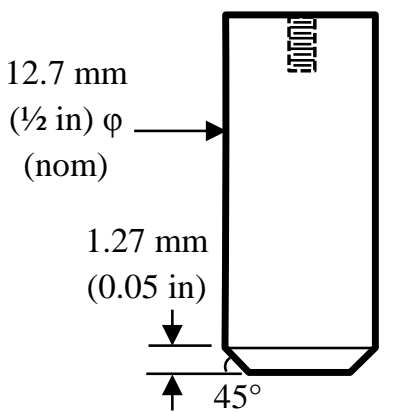

b. Flat peg

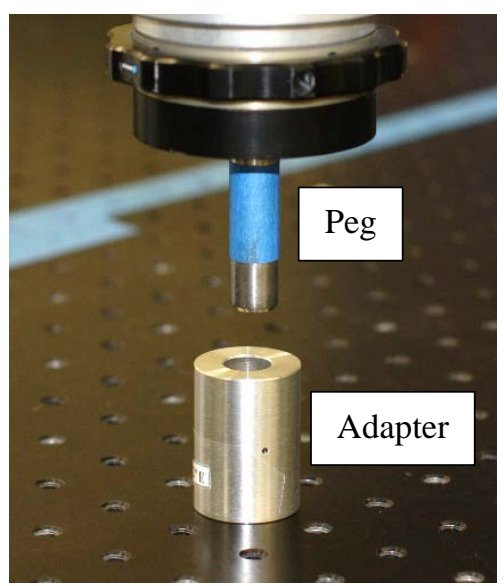

c. Flat peg and aluminum adapter

Figure 1. Conical and flat pegs and aluminum adapter. Note: (a) and (b) are not drawn to scale.

The experiments were conducted using a surface consisting of six, $600 \mathrm{~mm}$ x $900 \mathrm{~mm}$, optical breadboard plates mounted on a rigid aluminum frame. Each plate has a flatness of $\pm 0.15 \mathrm{~mm}$ over $0.09 \mathrm{~m}^{2}$. The experiments were conducted using a single plate to reduce any additional noise in the measurements from misalignment or offset of the plates. The plates had threaded holes spaced at $25 \mathrm{~mm}$ (0.98 in). The nominal locations of the holes were taken as ground truth locations. The work volume is essentially $2 \mathrm{D}$ and consisted of 17 rows by 21 columns of holes (357 holes) - see Figure 2. The adapters were screwed into the threaded holes in the optical table. 


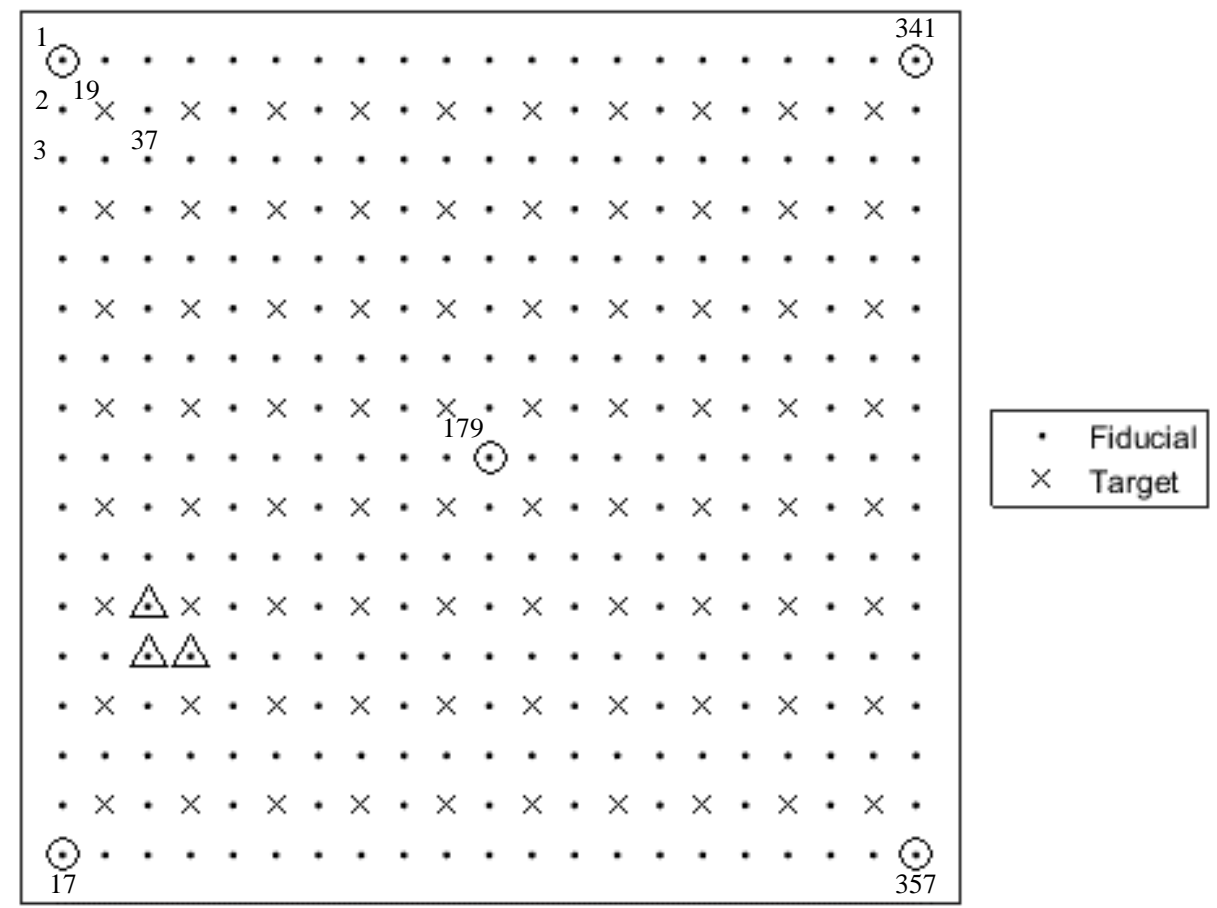

Figure 2. Layout of the $17 \times 21$ grid of threaded holes used in the experiments. The circles are fiducials used for conventional registration and triangles are fiducials used for registration in the RRBC method.

\subsection{TEST PROCEDURE}

\subsubsection{General procedure and concept of experiments}

As mentioned in Section 2.1, the ground truth locations (in world coordinate frame) of the holes were taken as the nominal locations of the holes in the world coordinate frame; thus, no measurements were needed. The locations of all holes, in robot coordinate frame, were obtained by manually inserting the peg into the adapter, and recording the $\left(x, y, z, \vartheta_{x}, \vartheta_{y}, \vartheta_{z}\right)$ values; these will be called the raw measured values. Each position was only measured once, i.e., no repeats. These manual measurements were obtained prior to the start of each experiment and used for the RRBC method.

Based on the raw measurements, corrections to restore the rigid body condition were then calculated as described in [6] for all fiducials. Since, the experiments in this report are essentially 2D, only corrections to the $x$ - and $y$-coordinates were calculated, and the $z$-coordinate was unchanged from the raw measured value. Also, corrections were only made to the position $(x, y, z)$, and no corrections were made to the angles. 
As seen in Figure 2, targets were located in every other column and every other row, resulting in a total of 80 targets. Being a research study, the locations of the targets were measured beforehand in the robot coordinate frame using manual measurements - this is not feasible in practice and target locations are usually unknown. However, to imitate practice, target locations were treated as unknown positions in the calculations, and the surrounding, manually-obtained fiducial points were used for the calculations using the RRBC method. Therefore, the correction to a target location was estimated by linearly interpolating the fiducial corrections of the eight fiducials closest to the target. ${ }^{2}$ The measured target locations (i.e., raw measured locations in robot frame) were only used to determine the registration error, TRE.

Once the corrections for the fiducials and targets were calculated, two transformations from the world coordinate frame (working frame) to the robot coordinate frame (destination frame) were determined. The first or uncorrected transformation was obtained by using the uncorrected fiducials (Points 1, 17 and 357 for Experiments 1 and 2, and Points 1, 17, 179, 341, and 357 for Experiment 3). The choice of the three fiducials in Experiments 1 and 2 was based on the common practice of selecting well-distributed fiducials. The addition of Points 179 and 341 in Experiment 3 was an effort to improve the performance of the uncorrected registration as the use of more fiducials reduces the registration error. The uncorrected transformation was then applied to all uncorrected fiducials and uncorrected targets; these transformed positions were called uncorrected positions.

The second or corrected transformation was obtained by using the corrected fiducials shown in Figure 2 as triangles. These fiducials would normally not be selected as it would be bad practice to select fiducials so close together. However, these fiducials were chosen, on purpose, to show that once the rigid-body condition is restored, it does not matter which corrected fiducials were selected for registration. The corrected transformation was then applied to all corrected fiducials and corrected targets (recall that the corrections to the targets are estimated based on the corrected neighboring fiducials); these transformed positions were called corrected positions.

A third set of positions, to which no transformation was applied, was also used. These positions were the raw measured positions of the targets. These insertions reflect the time-consuming process of lead through programming and should always pass (i.e., be fully inserted) as these were the manually-measured positions in the robot coordinate frame. The performance using these positions served as a baseline.

The format of the input files was $\left(x, y, z, \vartheta_{x}, \vartheta_{y}, \vartheta_{z}\right)$ for all fiducial and/or target positions where $(x, y, z)$ were either the raw, uncorrected, or corrected positions and $\left(\vartheta_{x}, \vartheta_{y}, \vartheta_{z}\right)$ were either Original angles (raw measured values for a given position) or Fixed angles (see Section 2.2.3).

\footnotetext{
${ }^{2}$ Note that this procedure is different than that described in Section 1 which pertains to a 3D situation.
} 
The basic experiment involved 1) uploading the various sets of coordinates (raw, uncorrected, corrected) to the robot controller, 2) having the robot move to the first position specified in the raw file, 3) inserting the peg into the hole, 4) extracting the peg, and 5) moving to a position slightly above the hole. These steps were then repeated at the same position using the uncorrected and corrected values. After the raw, uncorrected, and corrected insertions were attempted, the robot moved to the next specified position and repeated the insertions until all specified positions were evaluated.

To determine if the method to improve the registration was effective, a Pass/Fail metric was used. A Pass meant that the robot was able to fully insert the peg into the hole with a force less than $17 \mathrm{~N}$; otherwise, it was considered a Fail. It was also considered to have failed if the peg was fully inserted, but jammed while extracting the peg. The threshold of $17 \mathrm{~N}$ was arrived at experimentally as various force levels were tried to determine a level which would not cause the peg to jam badly (i.e., hard to extricate peg and the robot joints locked up).

Three experiments, Experiments 1 to 3, were conducted with slight variations from the procedure described above. These variations were based on observations and experience gained from the previous experiments and are described in the following subsections.

\subsubsection{Experiment 1, Runs 1-7}

Experiment 1 was repeated seven times using the conical peg. A repeat was a run through all the positions specified in the input files. For all runs (Runs $1-7$ ), the same adapter was used to reduce variability in the results due to the use of different adapters. All seven runs were completed in four days.

For Runs 1 - 3, the peg was inserted into all 357 positions (i.e., all fiducial and target locations). The input files for these runs were the uncorrected and corrected positions. The angles used were the Original angles.

The time to complete a run for all 357 positions took several hours. Since the information for target locations were of primary interest and to reduce the time for each run, the peg insertions were only performed for the 80 target locations for Runs 4 - 7. The input files for these runs were the raw, uncorrected, and corrected positions. The angles used were the Original angles.

\subsubsection{Experiment 2, Runs 9-15}

Three changes were made for Experiment 2. The first change was the use of two sets of angles. In Experiment 1, Original angles were used for all runs. To try to reduce the incidences of jams, it was felt that the use of the average angles (values averaged over all 357 measured raw positions) as opposed to the instantaneous measurement at each position would remove the effect

of noise in the angle measurement. Therefore, a second set angles, Fixed angles $=\left(\vartheta_{x_{-} a v g}, \vartheta_{y_{-} a v g}\right.$, 
$\vartheta_{z}$ ), was used for all positions where $\vartheta_{x_{\_} a v g}$ was the average of all $\vartheta_{x}$, similarly for $\vartheta_{y_{-} a v g}$. The value for $\vartheta_{z}$ was the same as that for the Original angle.

The second change was the use of another method to improve registration. In addition to the raw, uncorrected, and corrected positions, positions based on a local registration were determined. For the local registration, three uncorrected fiducials close to a target were used to determine the transformation from the working frame to the destination frame. For example, in Figure 2, the uncorrected target (point 19) was transformed by using uncorrected fiducial points 1, 3, and 37. This transformation was only applied to that particular uncorrected target, point 19. This process was repeated for all targets, i.e., each target had a unique transformation. This local registration was expected to yield better registration results compared to the registration for the uncorrected positions which used one set of fiducials (Points 1, 17, and 357) to determine the transformation for all targets.

The third change was the use of a flat peg instead of the conical peg used in Experiment 1 as explained in Section 2.1.

Experiment 2 was repeated seven times using the raw, uncorrected, corrected, and local registration positions and the Original angles, and seven times using the raw, uncorrected, corrected, and local registration positions and Fixed angles. For these 14 runs, insertions were performed for 80 targets only, and it took four days to complete all the runs.

The same adapter used in Experiment 1 was used in Experiment 2 except for Run 15, Original angles. A new adapter was used for this run as the number of fails appeared to increase with time, and a possible explanation for this increase was that the adapter was getting damaged by repeated testing. However, the results from Run 15, Original angles showed that it was not the adapter causing the increase in the number of Fails with time.

\subsubsection{Experiment 3, Runs $16-27$}

Based on the preliminary results from Experiment 2, it appeared that the number of Fails increased with time, i.e., more Fails in the later runs. It was speculated that turning the robot on/off may be causing the robot origin to shift or that when the robot jammed, the joints would be slightly shifted. Since we could not control the latter, for Experiment 3, we tried to complete at least seven runs without turning the robot off.

To accomplish these continuous runs, streamlining the test procedure was essential. It was decided that insertions for the local positions would not be performed as preliminary findings showed that there was only a slight improvement with this method as compared to the uncorrected positions. Seven adapters were used instead of one adapter so that there would be little of no down time between insertions to move the adapter from one physical location to another. The hole diameters of the seven adapters varied between $12.7 \mathrm{~mm}$ and $12.7245 \mathrm{~mm}$ 
(0.500 in and 0.501 in). Finally, since there appeared to be fewer jams with the Fixed angles, only Fixed angles were used in Experiment 3.

There was also a change to the registration process for the uncorrected targets which was to use five fiducials instead of three as was done in Experiments 1 and 2. The expected effect of using more fiducials was a reduced number of failures for the uncorrected positions as past studies have indicated that an increased number of fiducials used for registration reduced the registration error. The number of fiducials used for the corrected positions remained at three.

Seven runs were completed without turning the robot off. However, the number of Fails increased in the later runs as observed in the previous experiments. It was decided to perform more runs to determine if the number of Fails would continue to increase, or if the failure rate would level off - the robot was turned on/off for the later runs. In all, a total of 12 runs were performed over a period of four days.

In summary, Experiment 3 consisted of 12 runs using the flat peg and the input positions were raw, uncorrected, and corrected for Fixed angles.

\subsection{NOISE MEASUREMENT}

Measurements of 52 targets were made to characterize the noise of the measurements. For each target position, 16 repeats were made. Each repeat consisted of the following steps: 1) screwing the adapter to the optical table, 2) manually inserting the peg by hand-guiding the robot into position, 3) recording the pose ( $\left.x, y, z, \vartheta_{x}, \vartheta_{y}, \vartheta_{z}\right)$ at the position, 4) hand-guiding the robot up to remove the peg, 5 ) unscrewing the adapter, and 6) moving the robot arm to a random position.

\section{RESULTS AND DISCUSSION}

\subsection{FAILURE RATE: CONVENTIONAL REGISTRATION VS. RRBC MEHOD}

For each run, the number of times the peg failed to be inserted was determined and the ratio of this number to the total number of insertions (277 for fiducials and 80 for targets) was calculated and plotted. The plots of the percent Fails for the three experiments are shown in Figure 3 Figure $6^{3}$. The data from these plots are given in Table 1 . In Table 1, gray cells indicate that no data was collected for that parameter. The columns labeled "Uncor. - Cor." quantify the improvement of the RRBC (corrected) method as compared to the conventional (uncorrected) registration method. The averages and standard deviations, $s$, of the data are also given in Table 1. In Figure 3 - Figure 6 and Table 1, the baseline values are the results for the raw insertions. As mentioned in Section 2.2.1, there should be no failures for the baseline insertions, and the

\footnotetext{
${ }^{3}$ Plots showing failed insertions at target locations for all runs and for all experiments are given in the Appendix.
} 
observed failures (about $2 \%$ to $6 \%$ ) are likely due to measurement noise as the raw measurements were based on single measurements and to robot drift.

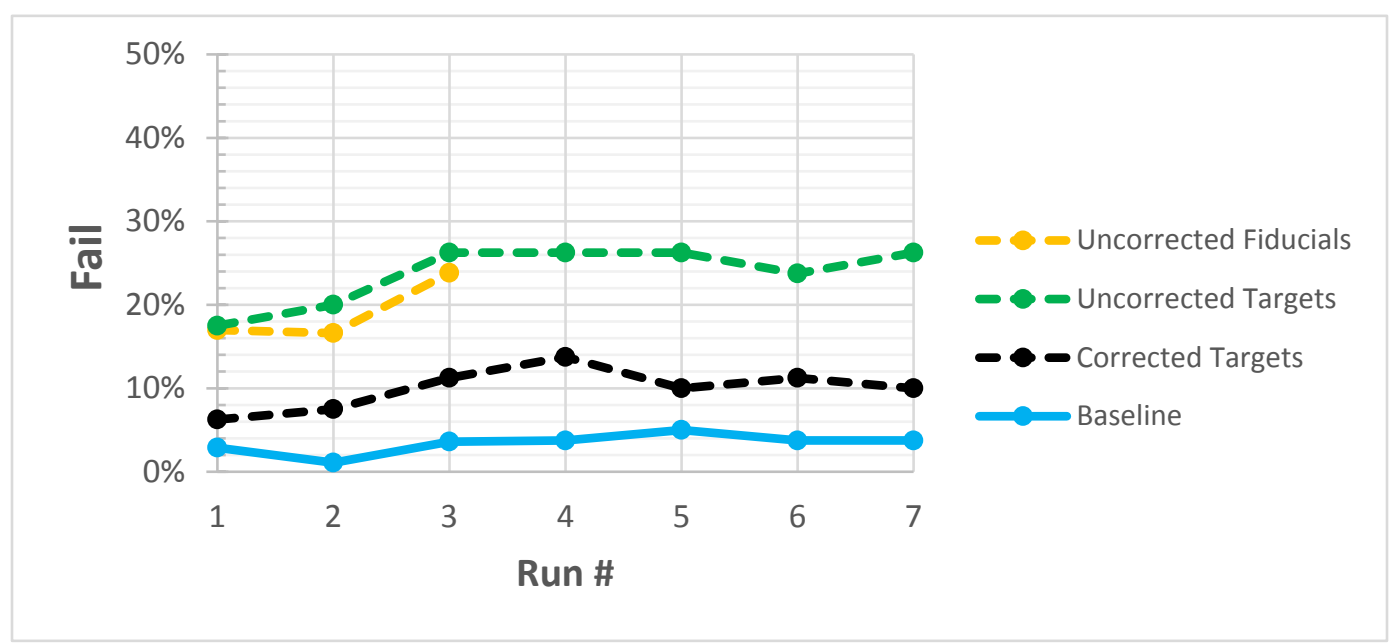

Figure 3. Experiment 1: Runs 1-7, Original angles, conical peg, fiducials and targets.

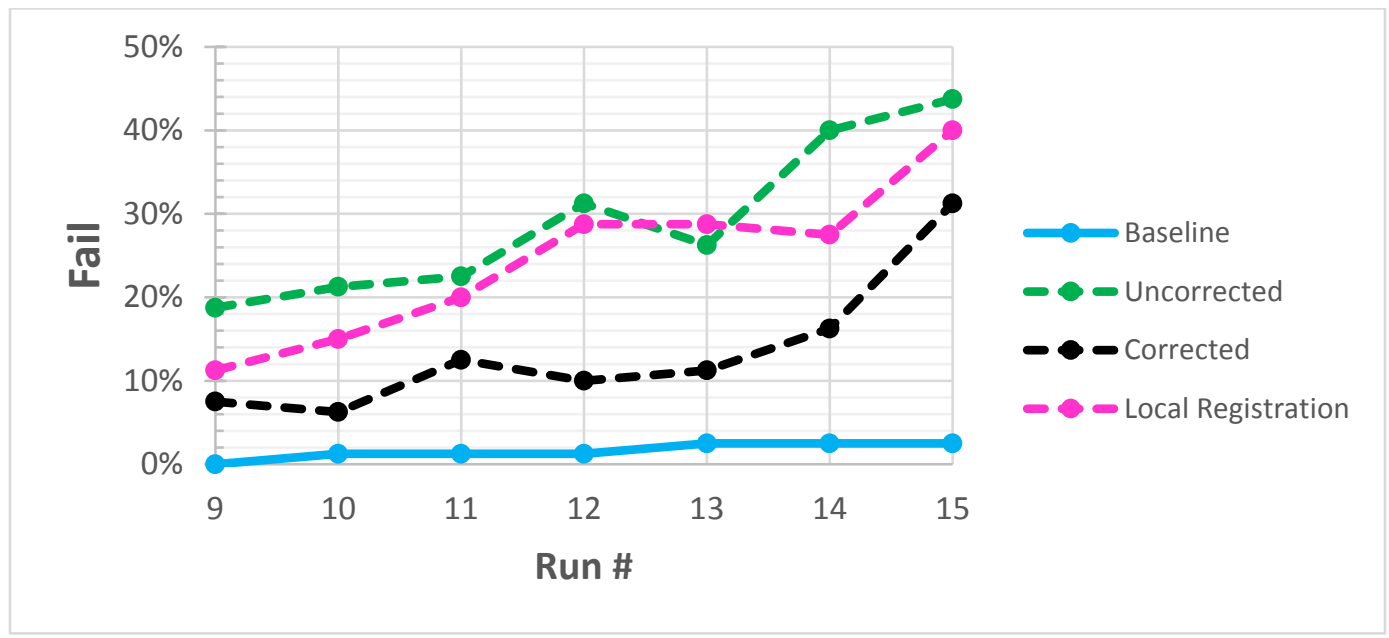

Figure 4. Experiment 2: Runs 9-15, Original angles, flat peg, targets only. 


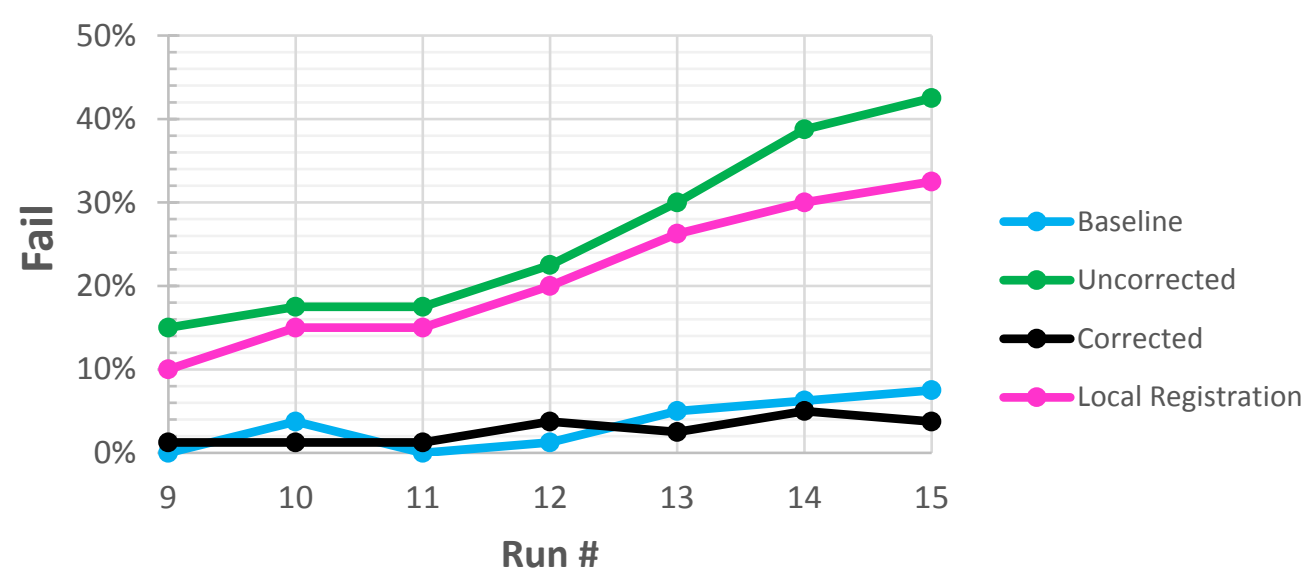

Figure 5. Experiment 2: Runs 9-15, Fixed angles, flat peg, targets only.

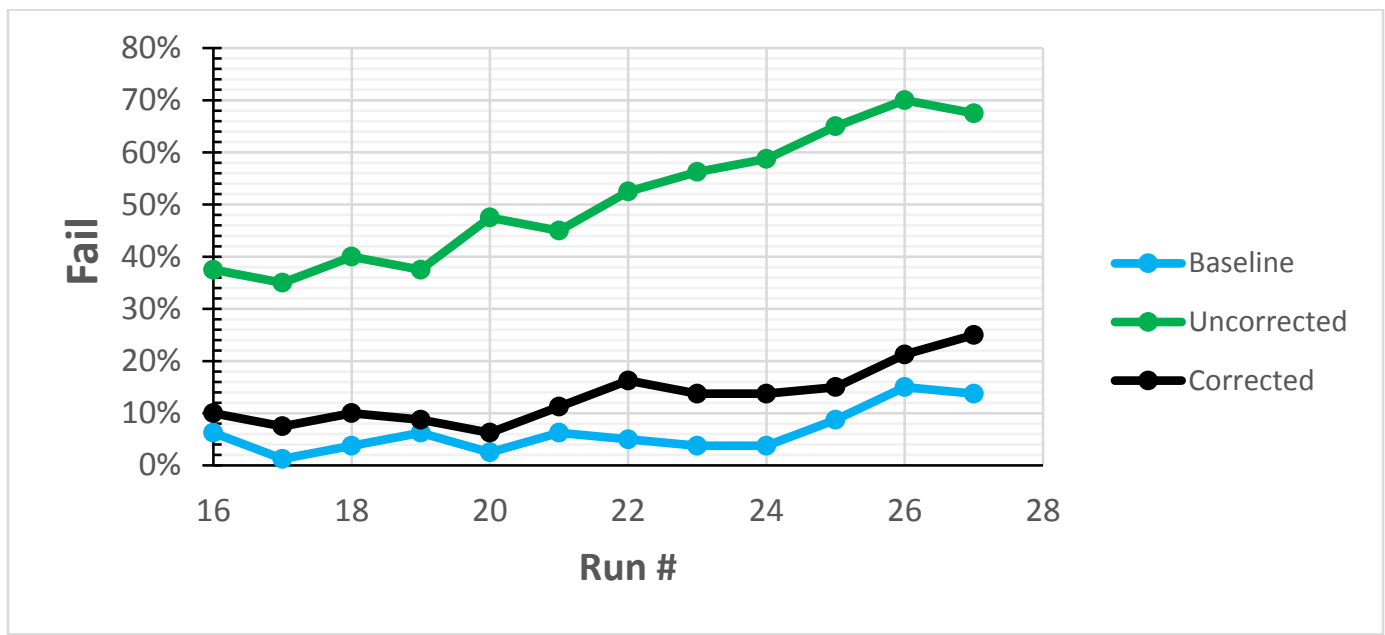

Figure 6. Experiment 3: Runs 16-27, Fixed angles, flat peg, targets only. 
Table 1. Percentage of Failed Insertions for Experiments 1 - 3, Targets only.

\begin{tabular}{|c|c|c|c|c|c|c|c|c|c|c|}
\hline \multirow[b]{3}{*}{ Run \# } & \multicolumn{10}{|c|}{ Failed Insertion (\%) } \\
\hline & \multicolumn{5}{|c|}{ Original Angles } & \multicolumn{5}{|c|}{ Fixed Angles } \\
\hline & Baseline & Uncor. & Cor. & $\begin{array}{l}\text { Local } \\
\text { Reg. }\end{array}$ & $\begin{array}{l}\text { Uncor. } \\
\text { - Cor. }\end{array}$ & Baseline & Uncor. & Cor. & $\begin{array}{l}\text { Local } \\
\text { Reg. }\end{array}$ & $\begin{array}{l}\text { Uncor } \\
\text { - Cor. }\end{array}$ \\
\hline \multicolumn{11}{|c|}{ EXPERIMENT 1} \\
\hline 1 & 2.9 & 17.5 & 6.3 & & 11.3 & & & & & \\
\hline 2 & 1.1 & 20.0 & 7.5 & & 12.5 & & & & & \\
\hline 3 & 3.6 & 26.3 & 11.3 & & 15.0 & & & & & \\
\hline 4 & 3.8 & 26.3 & 13.8 & & 12.5 & & & & & \\
\hline 5 & 5.0 & 26.3 & 10.0 & & 16.3 & & & & & \\
\hline 6 & 3.8 & 23.8 & 11.3 & & 12.5 & & & & & \\
\hline 7 & 3.8 & 26.3 & 10.0 & & 16.3 & & & & & \\
\hline Avg. & 3.4 & 23.8 & 10.0 & & 13.8 & & & & & \\
\hline$s$ & 1.2 & 3.6 & 2.5 & & 2.0 & & & & & \\
\hline \multicolumn{11}{|c|}{ EXPERIMENT 2} \\
\hline 9 & 0.0 & 18.8 & 7.5 & 11.3 & 11.3 & 0.0 & 15.0 & 1.3 & 10.0 & 13.8 \\
\hline 10 & 1.3 & 21.3 & 6.3 & 15.0 & 15.0 & 3.8 & 17.5 & 1.3 & 15.0 & 16.3 \\
\hline 11 & 1.3 & 22.5 & 12.5 & 20.0 & 10.0 & 0.0 & 17.5 & 1.3 & 15.0 & 16.3 \\
\hline 12 & 1.3 & 31.3 & 10.0 & 28.8 & 21.3 & 1.3 & 22.5 & 3.8 & 20.0 & 18.8 \\
\hline 13 & 2.5 & 26.3 & 11.3 & 28.8 & 15.0 & 5.0 & 30.0 & 2.5 & 26.3 & 27.5 \\
\hline 14 & 2.5 & 40.0 & 16.3 & 27.5 & 23.8 & 6.3 & 38.8 & 5.0 & 30.0 & 33.8 \\
\hline 15 & 2.5 & 43.8 & 31.3 & 40.0 & 12.5 & 7.5 & 42.5 & 3.8 & 32.5 & 38.8 \\
\hline Avg. & 1.6 & 29.1 & 13.6 & 24.5 & 15.5 & 3.4 & 26.3 & 2.7 & 21.3 & 23.6 \\
\hline$s$ & 0.9 & 9.6 & 8.5 & 9.8 & 5.1 & 3.0 & 11.0 & 1.5 & 8.5 & 9.8 \\
\hline \multicolumn{11}{|c|}{ EXPERIMENT 3} \\
\hline 16 & & & & & & 6.3 & 37.5 & 10.0 & & 27.5 \\
\hline 17 & & & & & & 1.3 & 35.0 & 7.5 & & 27.5 \\
\hline 18 & & & & & & 3.8 & 40.0 & 10.0 & & 30.0 \\
\hline 19 & & & & & & 6.3 & 37.5 & 8.8 & & 28.8 \\
\hline 20 & & & & & & 2.5 & 47.5 & 6.3 & & 41.3 \\
\hline 21 & & & & & & 6.3 & 45.0 & 11.3 & & 33.8 \\
\hline 22 & & & & & & 5.0 & 52.5 & 16.3 & & 36.3 \\
\hline 23 & & & & & & 3.8 & 56.3 & 13.8 & & 42.5 \\
\hline 24 & & & & & & 3.8 & 58.8 & 13.8 & & 45.0 \\
\hline 25 & & & & & & 8.8 & 65.0 & 15.0 & & 50.0 \\
\hline 26 & & & & & & 15.0 & 70.0 & 21.3 & & 48.8 \\
\hline 27 & & & & & & 13.8 & 67.5 & 25.0 & & 42.5 \\
\hline Avg. & & & & & & 6.4 & 51.0 & 13.2 & & 37.8 \\
\hline$s$ & & & & & & 4.2 & 12.5 & 5.6 & & 8.3 \\
\hline
\end{tabular}

The most obvious observation from Figure 3 - Figure 6 is that the RRBC improves the conventional registration method. The average improvement was approximately $15 \%$ for the insertions with the Original angles (Exp. 1 and 2) and approximately $24 \%$ and $38 \%$ for the Fixed angles, Exp. 2 and 3, respectively.

The other noticeable trend from the figures, especially with the later runs, is the increase in the number of failures with time. Some possible reasons for this increase were thermal effects as the 
day wore on and turning the robot on/off each day. However, the thermal effects would not explain a similar or larger number of failures for the last run from one day compared the first run at the start of the next day. That is, if thermal effects were an issue, there should be fewer failures at the beginning of the next day. Since the coordinates to which the robot was commanded to move were unchanged for all runs, it appeared that turning the robot on/off every day may cause the origin of the robot be slightly different each time. Therefore, it was decided to conduct a third experiment. As explained in Section 2.2.4, the first seven runs in Experiment 3 (Runs 16-22) were conducted continuously in one shift without turning the robot off. As seen in Figure 6, Runs 16-22, the number of failures still increased with time. At this time, the only explanation that can be offered for this increased number of failures is that the robot, sensors, and mechanics may drift with time. Since turning the robot on and off did not seem to significantly affect the robot performance, it was decided to conduct more runs to determine if the number of failures would level off. As seen in Figure 6, the number of failures did not seem to be leveling off, and after 12 runs the percent of failures was about $70 \%$. In practice, plots similar to those shown in Figure 3 to Figure 6 could be developed for different hole tolerances to help practitioners in determining when a re-registration would be needed to reduce the number of failures.

The increased number of failures with time is more obvious for the flat peg than for the conical peg. Due to its shape, the conical peg can inherently accommodate more misalignment, and thus, experiences a lower number of failures. Comparisons of the slopes for the conventional and the RRBC methods in Figure 5 and Figure 6 show that the failure rate over time is greater for the conventional method.

A comparison of the seven runs in Experiment 2 and to the first seven runs in Experiment 3 shows that the percent of failures for the uncorrected method for Experiment 3 was higher than that for Experiment 2: an average of $26 \%$ vs. $42 \%$ for Experiments 2 and 3, respectively. This contradicts the expectation that the use of five fiducials (Experiment 3) would result in a better registration than the registration with three fiducials (Experiment 2). A reason for the higher rate of failures for the uncorrected method in Experiment 3 was because the diameter of the hole in the adapter used in Experiment 2 was between $12.751 \mathrm{~mm}$ and $12.776 \mathrm{~mm}$ (0.502 in and 0.503 in) while the diameters of the holes in the adapters used for Experiment 3 ranged from 12.700 $\mathrm{mm}$ and $12.725 \mathrm{~mm}$ (0.500 in and $0.501 \mathrm{in})$. The diameter of the flat peg was $12.675 \mathrm{~mm}$ (0.499 in) at the start of Experiment 2 and $12.662 \mathrm{~mm}$ (0.4985 in) after Experiment 3. Thus, the higher failure rate for the conventional method, an increase of about $16 \%$, in Experiment 3 can be attributed to the tighter tolerance - a decrease of $0.025 \mathrm{~mm}(0.001 \mathrm{in})$. The failure rate may even be higher as it was expected that the use of five fiducials would reduce the failure rate in Experiment 3. The tighter tolerance increased the failure percentage from $3 \%$ to $10 \%\left(1^{\text {st }}\right.$ seven runs Exp. 3) for the RRBC method.

Another observation is that in Experiment 3, the percent of failed insertions for the uncorrected method started out at about $37 \%$. Since the average baseline value was about $6 \%$ (i.e., $6 \%$ is 
due to noise), $31 \%$ of the Fails was due to the registration error. This is 31 failures for every 100 insertions, and this rate of failure may be unacceptable for many applications. Thus, for insertions where tight tolerances are needed, the RRBC method may have to be used as the failure rate started at about $(10 \%-6 \%)=4 \%$ or 4 failures for every 100 insertions.

The discussion above is based on the failed insertions in a given run. Another way to view the data is to look at the failed positions. A position is considered to have failed in Experiments 1 and 2 if four or more runs had failed insertions at that position and is considered to have failed in Experiment 3 if seven or more runs had failed insertions at that position; that is, the position failed for greater than $50 \%$ of the runs. This method reduces the effect of noise as it considers the failed insertions over 7 or 12 runs for a given position. A summary of the failed positions is given in Table 2. As seen in the table, the RRBC method improved the conventional registration method. The improvement for Original angles is about 15 \% (Exp. 1 and 2). For Fixed angles, the improvement for the tighter tolerances is about 33 \% (Exp. 3) compared with 22 \% (Exp. 2) for a looser tolerance. The analyses based on failed positions yield very similar results to the analyses based on failed insertions.

Table 2. Failed Target Positions.

\begin{tabular}{|c|c|c|c|c|c|c|c|c|c|c|}
\hline & \multicolumn{10}{|c|}{ Failed Positions } \\
\hline & \multicolumn{5}{|c|}{ Original Angles } & \multicolumn{5}{|c|}{ Fixed Angles } \\
\hline & Baseline & Uncor. & Cor & $\begin{array}{l}\text { Local } \\
\text { Reg. }\end{array}$ & $\begin{array}{l}\text { Uncor. } \\
\text { - Cor. }\end{array}$ & Baseline & Uncor. & Cor. & $\begin{array}{l}\text { Local. } \\
\text { Reg. }\end{array}$ & $\begin{array}{l}\text { Uncor. } \\
\text { - Cor. }\end{array}$ \\
\hline \multicolumn{11}{|c|}{ EXPERIMENT 1} \\
\hline $\begin{array}{c}\text { \% Failed Pos., } \\
7 \text { Runs }\end{array}$ & 3.8 & 25.0 & 10.0 & & 15.0 & & & & & \\
\hline \multicolumn{11}{|c|}{ EXPERIMENT 2} \\
\hline $\begin{array}{c}\text { \% Failed Pos., } \\
7 \text { Runs }\end{array}$ & 1.2 & 27.5 & 11.3 & 22.5 & 16.2 & 0 & 25.0 & 2.5 & 17.5 & 22.5 \\
\hline \multicolumn{11}{|c|}{ EXPERIMENT 3} \\
\hline $\begin{array}{c}\text { \% Failed Pos., } \\
12 \text { Runs }\end{array}$ & & & & & & 2.5 & 48.8 & 12.5 & & 36.3 \\
\hline $\begin{array}{c}\text { \% Failed Pos., } \\
1^{\text {st }} 7 \text { Runs }\end{array}$ & & & & & & 2.5 & 38.8 & 6.3 & & 32.5 \\
\hline
\end{tabular}

\subsection{LOCAL REGISTRATION METHOD}

As seen in Figure 4 and Figure 5, the local registration method only showed a small improvement (about 5 \% fewer Fails, see Table 1) over the conventional method. The improvement was expected but the amount of improvement was a little surprising. A study by Van Wyk and Marvel [8] using a pneumatic parallel gripper attached to the same 6DOF robot arm as used in this study showed an improvement of about $50 \%$ for a clustering method which is similar to the local registration method. The clustering method grouped the fiducials into $N$ clusters, and the transformation of a target was based on determining $\{\boldsymbol{R}, \boldsymbol{\tau}\}$ using the three 
fiducials closest to the target chosen from the fiducials in the cluster where the target resides. The peg used in [8] was a conical peg similar to the one used in Experiment 1. Possible reasons for the larger improvement in [8] may be due to noisier measurements from the use of 3D printed adapters with larger hole tolerances used in that study and the use of the pneumatic gripper with a 3D printed "wrist".

\subsection{ORIGINAL VS. FIXED ANGLES}

From Table 1, Experiment 2, a comparison of Original vs. Fixed angles shows that the use of Fixed angles had a greater improvement $-24 \%$ vs. $15 \%$. The comparison between Original vs. Fixed angles may be more easily seen in Figure 7 . The observed improvement was likely due to the reduced measurement noise as the Fixed angles averaged the angles over all 357 measured positions.

The use of Fixed angles does not impact the time to set-up/data collection task as the values of the Fixed angles are simply the averages of all the measured angles of the targets or if only fiducials were measured, the average angles for the fiducials may be used.

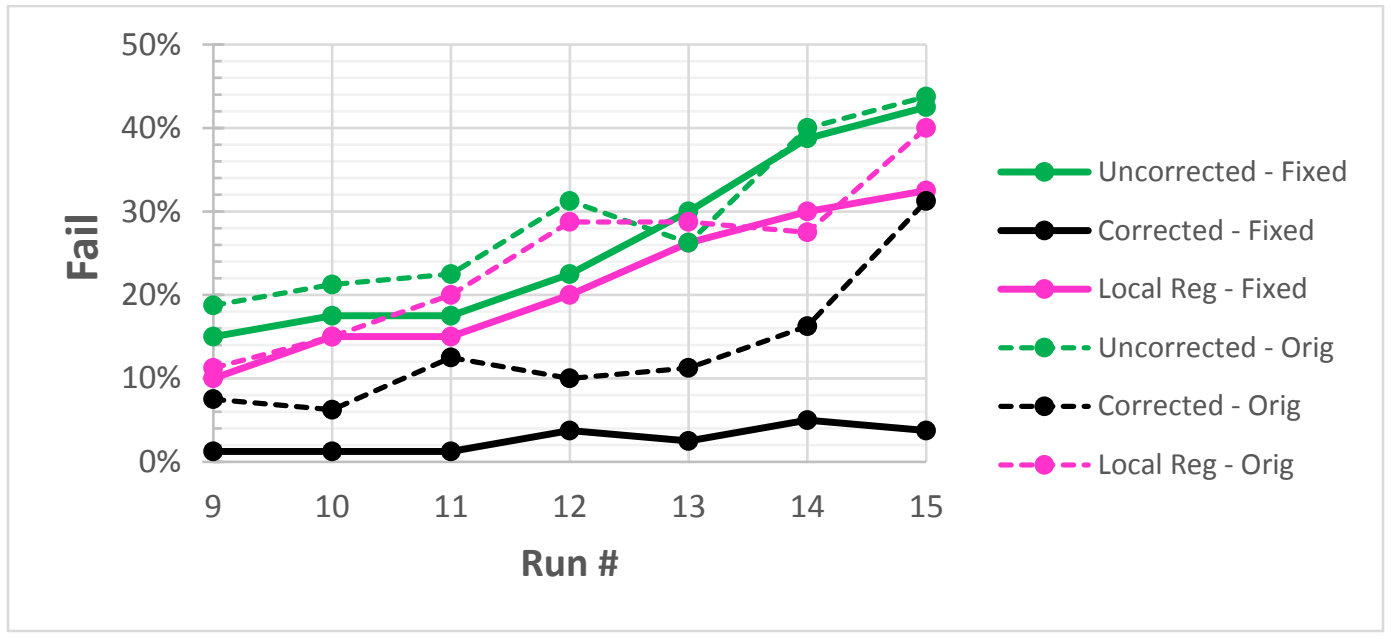

Figure 7. Experiment 2: Comparison of Original vs. Fixed angles. 


\section{$3.4 R^{R M S} S_{T}$}

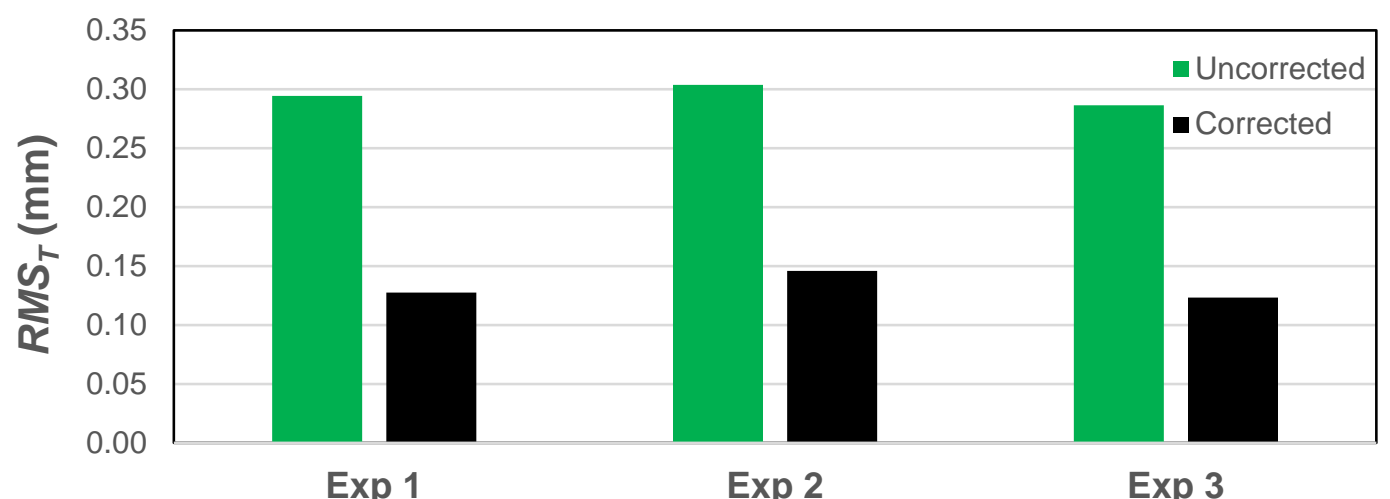

Figure 8. Comparison of $R M S_{T}$ for Experiments $1-3$.

The $R M S_{T}$ as calculated using Eq. 2, is shown in Figure 8. As noted earlier, a smaller $R M S_{T}$ indicates a better registration. The average reduction of the $R M S_{T}$ is about $60 \%$ for the RRBC method compared to the conventional method. This value is in line with the average values of $30 \%$ and $70 \%$ obtained for the two systems studied in [6].

\subsection{TARGET REGISTRATION ERROR (TRE)}

The TRE values for all targets were calculated using Eq. 3 and are summarized in Table 3. The differences between the TRE values for the conventional and RRBC methods are plotted against normalized failure values (see Figure 9 - Figure 12). The normalized failure value is the difference between the uncorrected failed insertions and corrected failed insertions divided by the number of runs for that experiment. A value greater than zero meant that the conventional method had more failed insertions and a value less than zero meant that the RRBC method had more failed insertions. The plots are divided into four quadrants:

1. Quad I (upper left) - RRBC increases TRE but reduces failures

2. Quad II (upper right) - RRBC reduces TRE and reduces failures

3. Quad III (lower left) - RRBC increases TRE and increases failures

4. Quad IV (lower right) - RRBC reduces TRE but increases failures

The results shown in Figure 9 - Figure 12 are summarized in Table 4. 
Table 3. Summary of TRE for all Experiments.

\begin{tabular}{|l|c|c|c|}
\hline & Exp. 1 & Exp. 2 & Exp. 3 \\
\hline Avg. Uncorrected TRE $(\mathrm{mm}) \quad[\mathrm{std} . \mathrm{dev}]$ & $0.255[0.148]$ & $0.267[0.146]$ & $0.278[0.135]$ \\
\hline Avg. Corrected TRE (mm) [std. dev] & $0.114[0.058]$ & $0.130[0.067]$ & $0.102[0.056]$ \\
\hline RRBC method reduced TRE (\%) & 55 & 51 & 63 \\
\hline
\end{tabular}

Table 4. Summary of Results Shown in Figure 9 - Figure 12.

\begin{tabular}{|c|c|c|c|c|c|}
\hline & \multicolumn{5}{|c|}{ Percentage of Points in the Different Quadrants } \\
\hline & Exp. 1, Orig. Angles & Exp. 2, Orig. Angles & Exp. 2, Fixed Angles & Exp. 3 Fixed Angles & Average \\
\hline $\mathrm{X}^{\mathrm{a}}>0$ & $88 \%$ & $85 \%$ & $85 \%$ & $93 \%$ & $88 \%$ \\
\hline Quad $1^{\mathrm{b}}$ & $0 \%$ & $0 \%$ & $1 \%$ & $3 \%$ & $1 \%$ \\
\hline Quad $2^{\mathrm{b}}$ & $25 \%$ & $43 \%$ & $48 \%$ & $75 \%$ & $48 \%$ \\
\hline Quad $3^{\mathrm{b}}$ & $4 \%$ & $5 \%$ & $1 \%$ & $1 \%$ & $3 \%$ \\
\hline Quad $4^{\mathrm{b}}$ & $3 \%$ & $6 \%$ & $1 \%$ & $4 \%$ & $3 \%$ \\
\hline
\end{tabular}




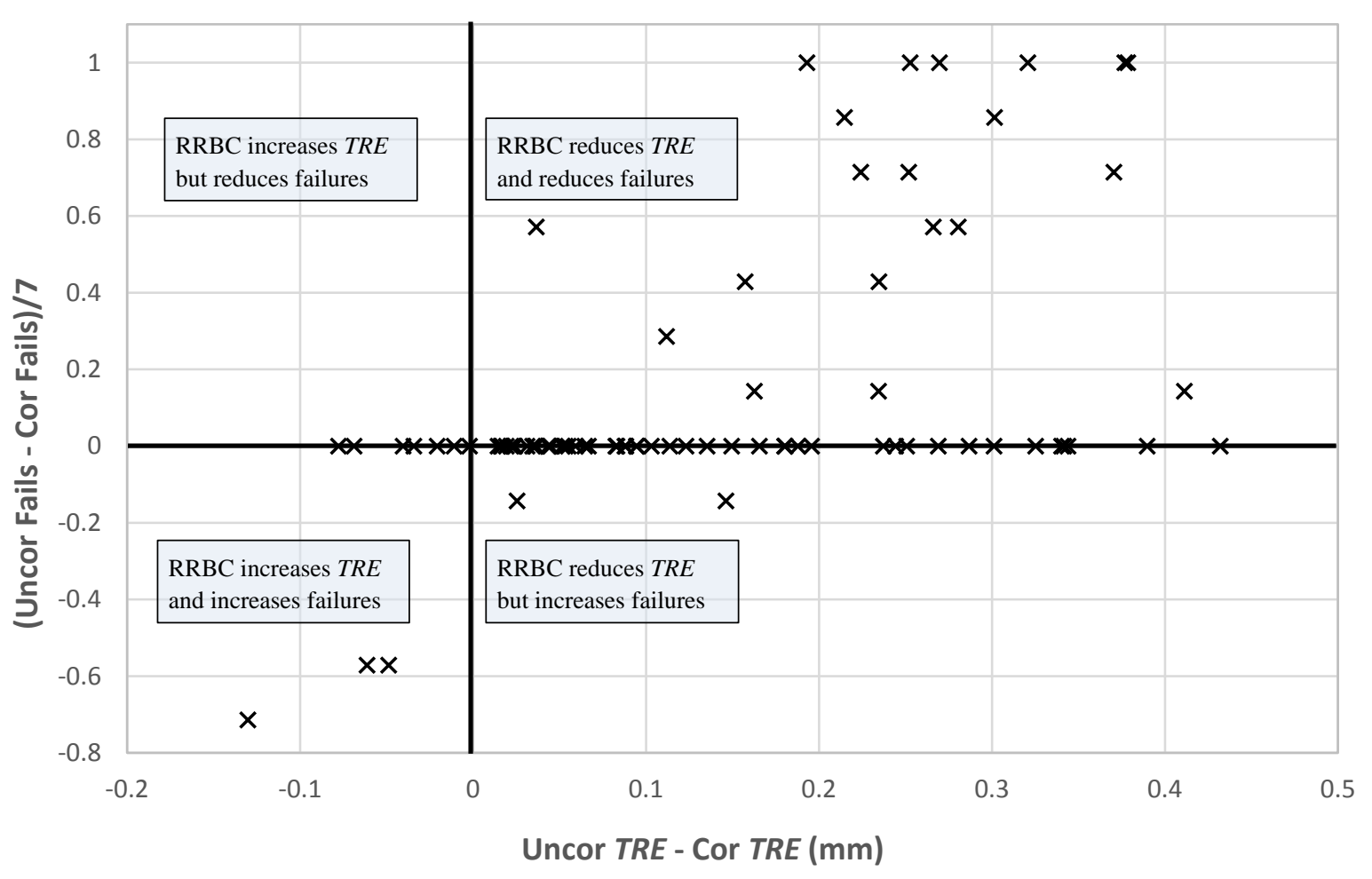

Figure 9 . Experiment 1, Original Angles.

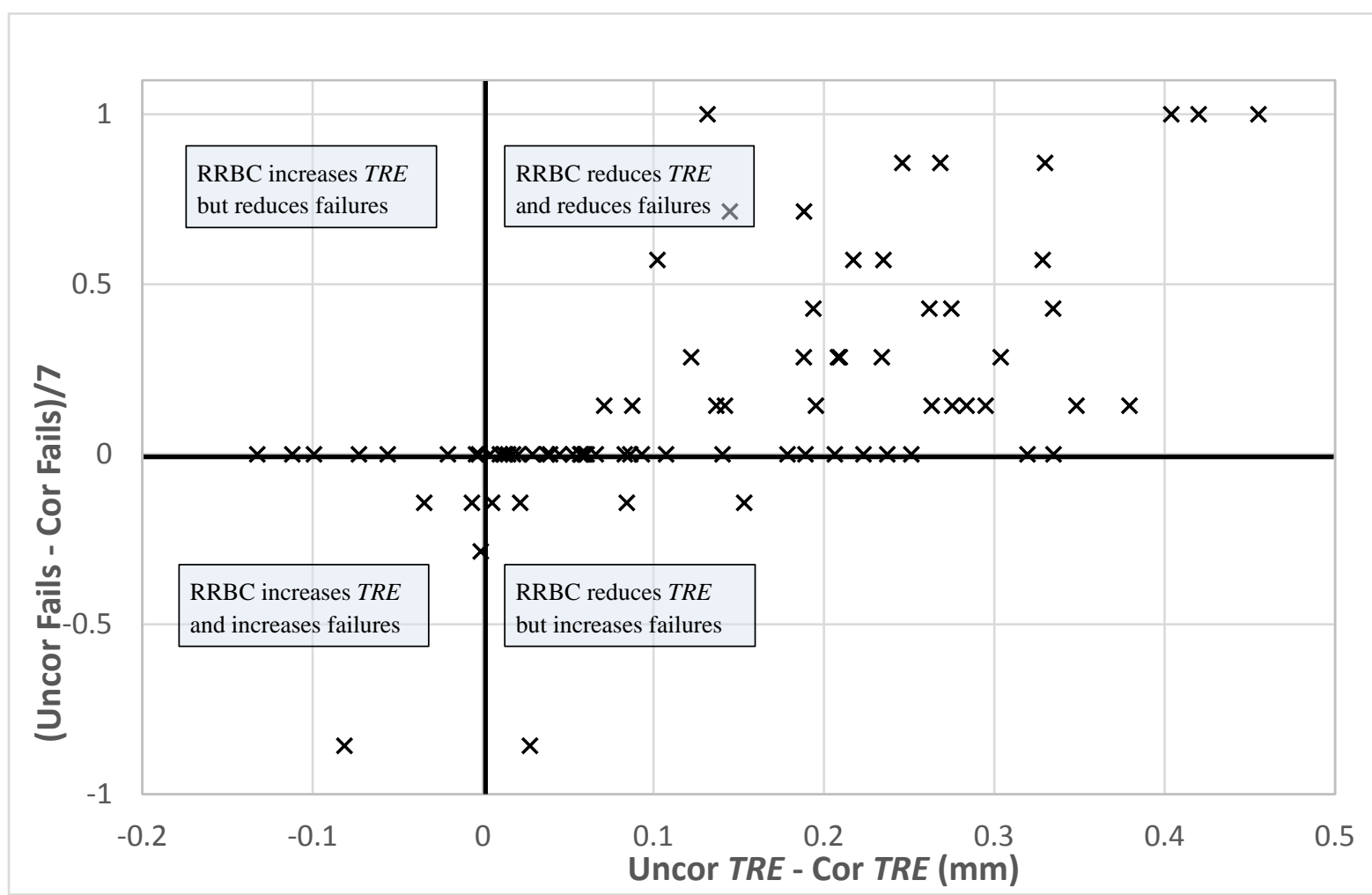

Figure 10. Experiment 2, Original Angles. 


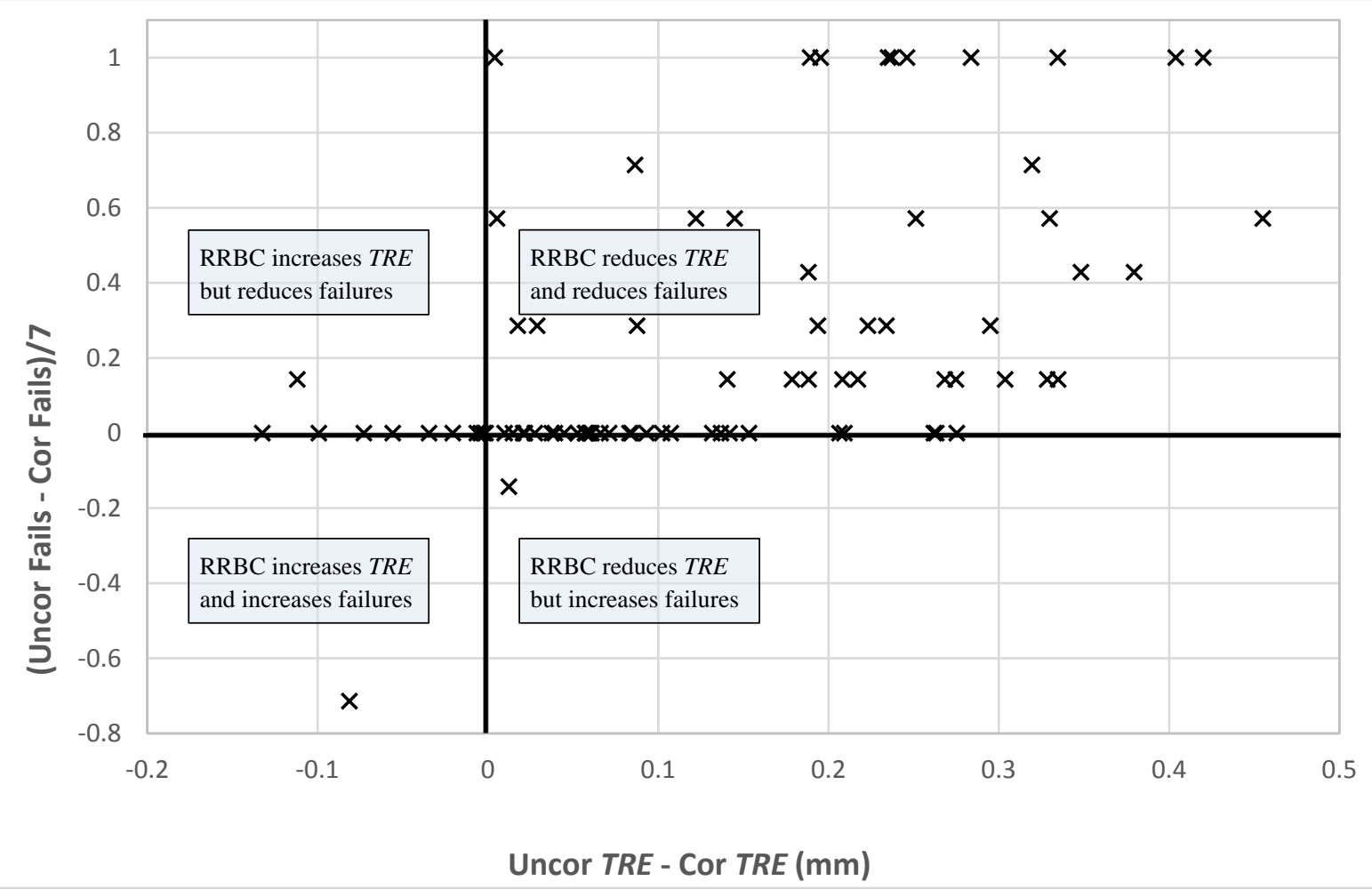

Figure 11. Experiment 2, Fixed Angles.

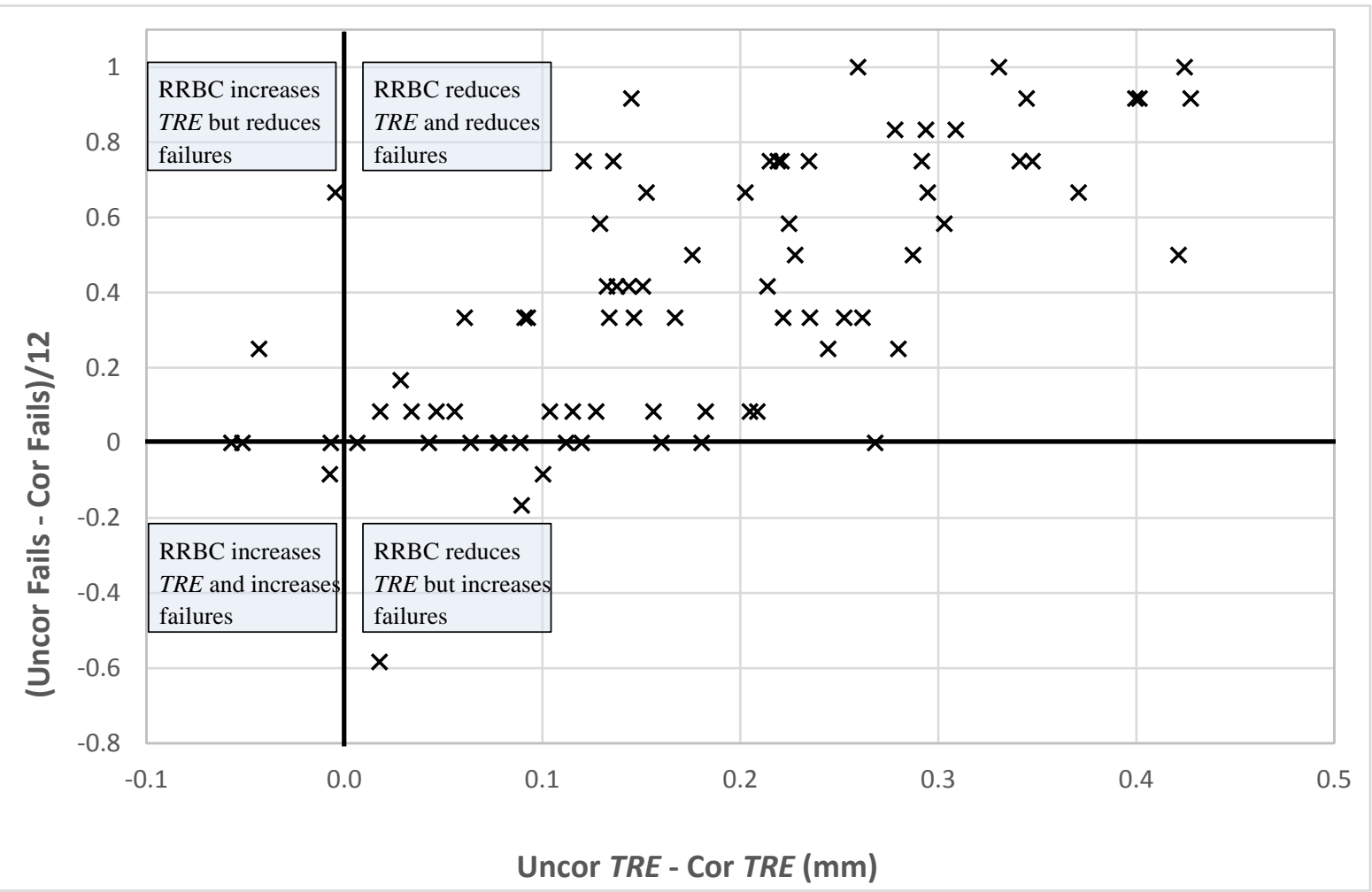

Figure 12. Experiment 3, Fixed Angles. 
As seen in Figure 9 - Figure 12 and Table 4, most of the points in all experiments are in Quad II and IV which indicate that the RRBC method reduced TRE and is an improvement over the conventional method. Also, excluding the points for which (Uncor Fails - Cor Fails) $/ 7=0$ (equal number of fails for conventional and RRBC methods), more points fall within Quad II indicating that RRBC reduces failures and is an improvement over the conventional method.

For Quads I, III, and IV, a couple of reasons are offered for why the RRBC method increases $T R E$ and/or increases failures. One reason is that the correction for the target is estimated based on the data from the neighboring points. The other reason is that linear interpolation is used to determine the corrections, i.e., the correction may not be linear.

It was expected that failed insertions/failed positions and TRE would be highly correlated higher occurrences of failed insertions at locations with higher TRE values. As seen in Figure 13 - Figure 16, this expected high correlation was not observed. However, there is a statistically significant positive correlation (95 \% confidence level, with p-values ranging from 0.002 to almost zero) between TRE and failed positions for the cases shown in Figure 13 - Figure 15. For Experiment 2, Corrected, Fixed angles (Figure 16), no conclusion could be drawn as there were too few failed positions. For Experiment 3, Corrected, Fixed angles (Figure 16), TRE was not significantly correlated with failed positions.

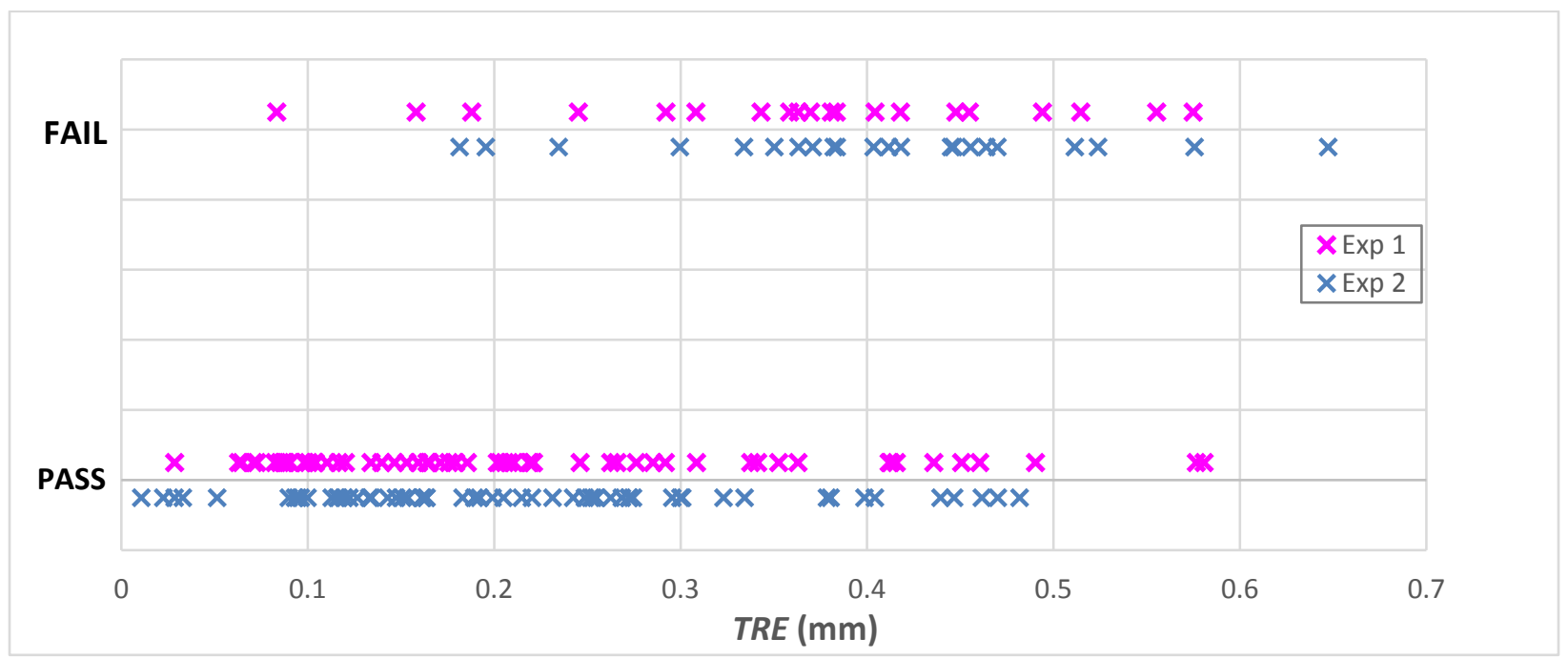

Figure 13. TRE vs. Failed Positions, Uncorrected, Original Angles. 


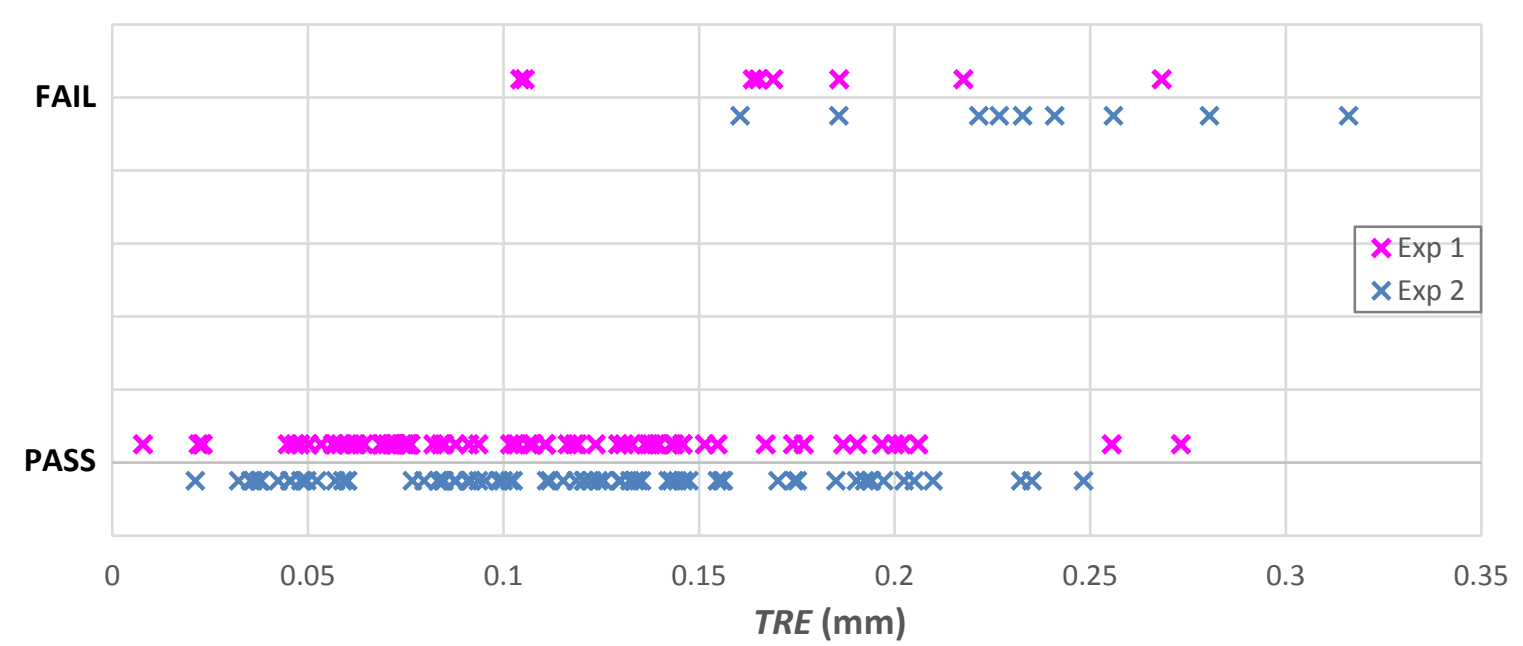

Figure 14. TRE vs. Failed Positions, Corrected, Original Angles.

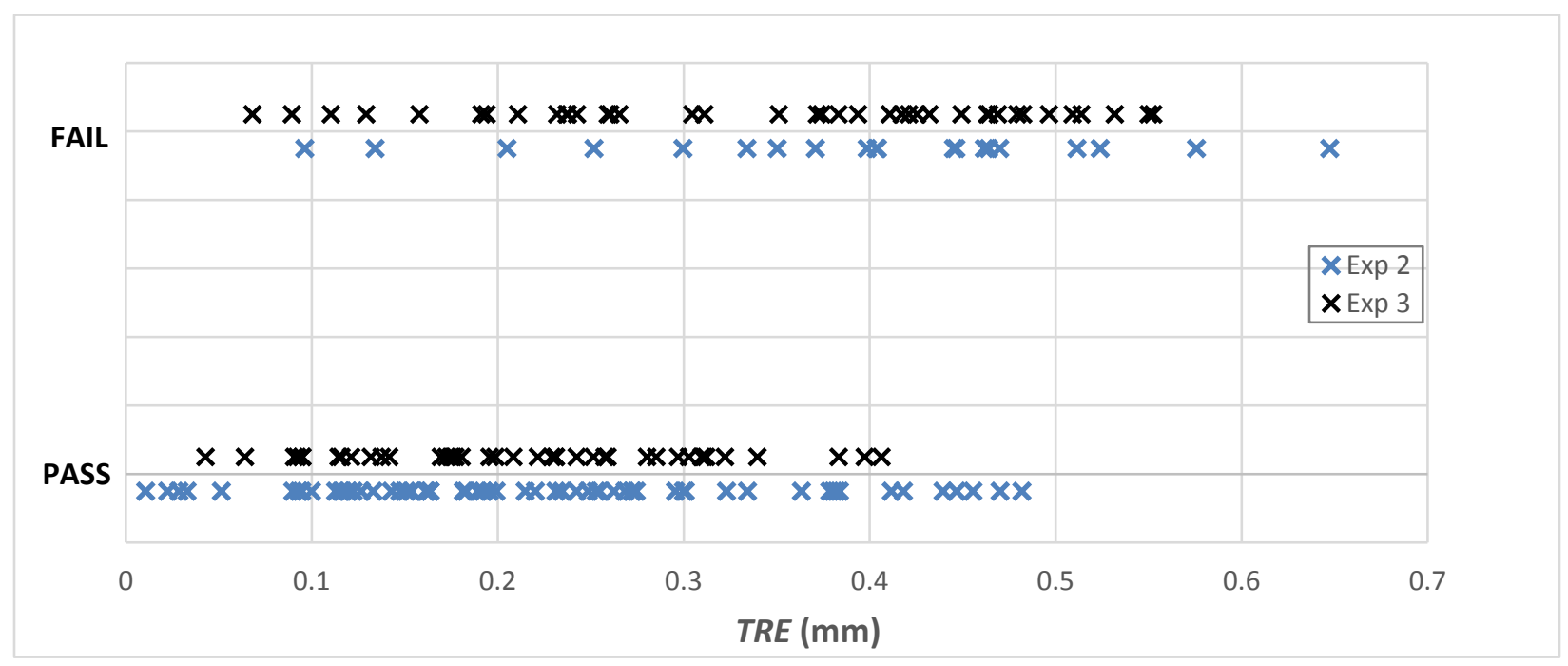

Figure 15. TRE vs. Failed Positions, Uncorrected, Fixed Angles. 


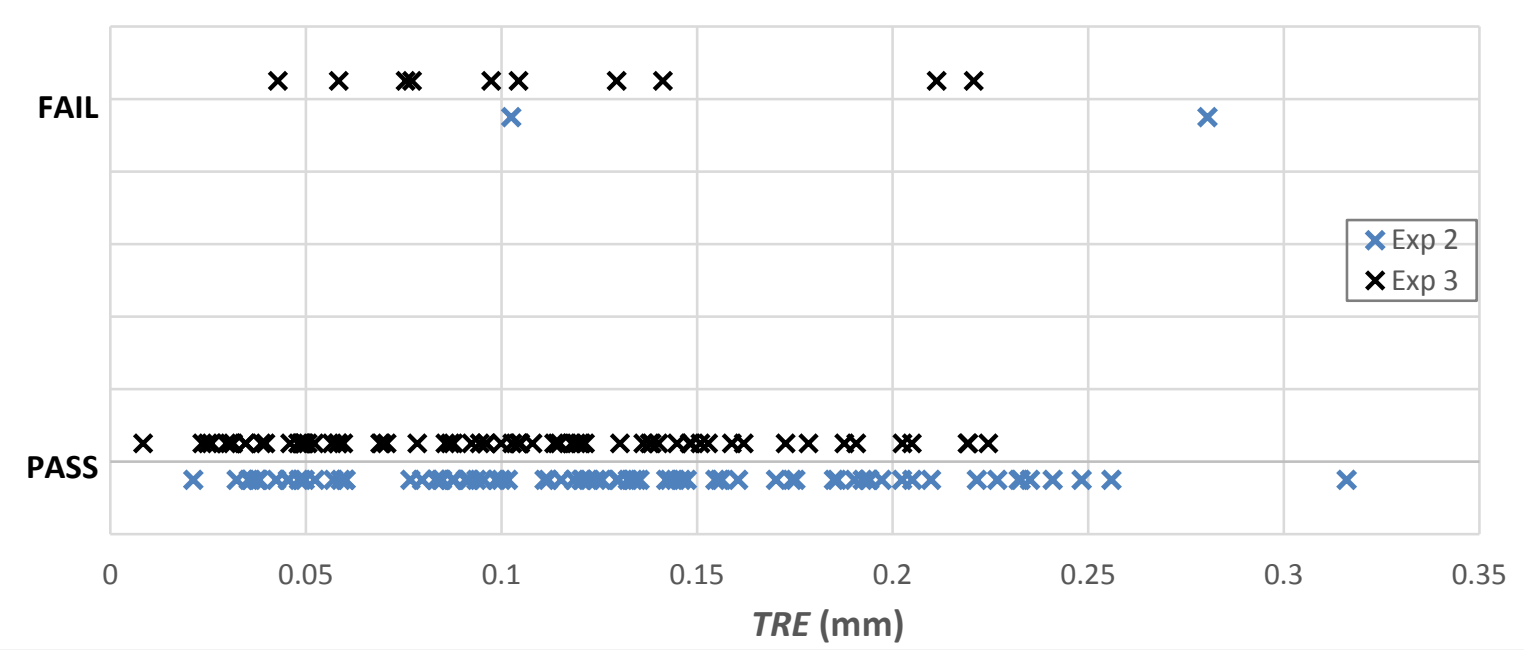

Figure 16. TRE vs. Failed Positions, Corrected, Fixed Angles.

\subsection{NOISE CHARACTERIZATION}

The positional noise, $\sigma_{n}$, was defined as:

$$
\sigma_{n}=\sqrt{\sigma_{x}^{2}+\sigma_{y}^{2}},
$$

where $\sigma_{x}$ and $\sigma_{y}$ are the standard deviations of the x- and y-coordinates of the target position. The z-component is omitted as these experiments were 2D. A plot of the positional noise for 52 targets are shown in Figure 17. The solid line in Figure 17 is the average noise which is about $0.113 \mathrm{~mm}$.

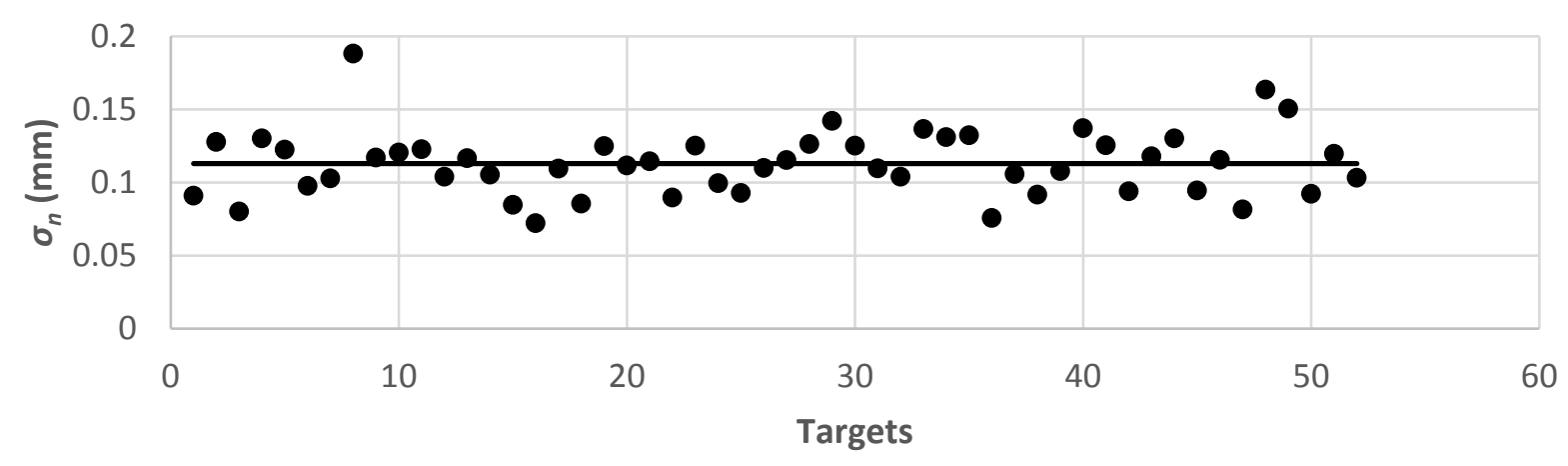

Figure 17. Positional Noise for 52 targets.

The standard deviations of the rotations are shown in Figure 18 where the solid lines represent the averages of the data. As seen in this figure, the standard deviations of rotation about the $\mathrm{x}$ - 
and $y$-axes are similar and higher than that for the z-axis. The lower value for the $\mathrm{z}$-axis is expected as the $\mathrm{z}$-axis coincides with the centerline of the peg.

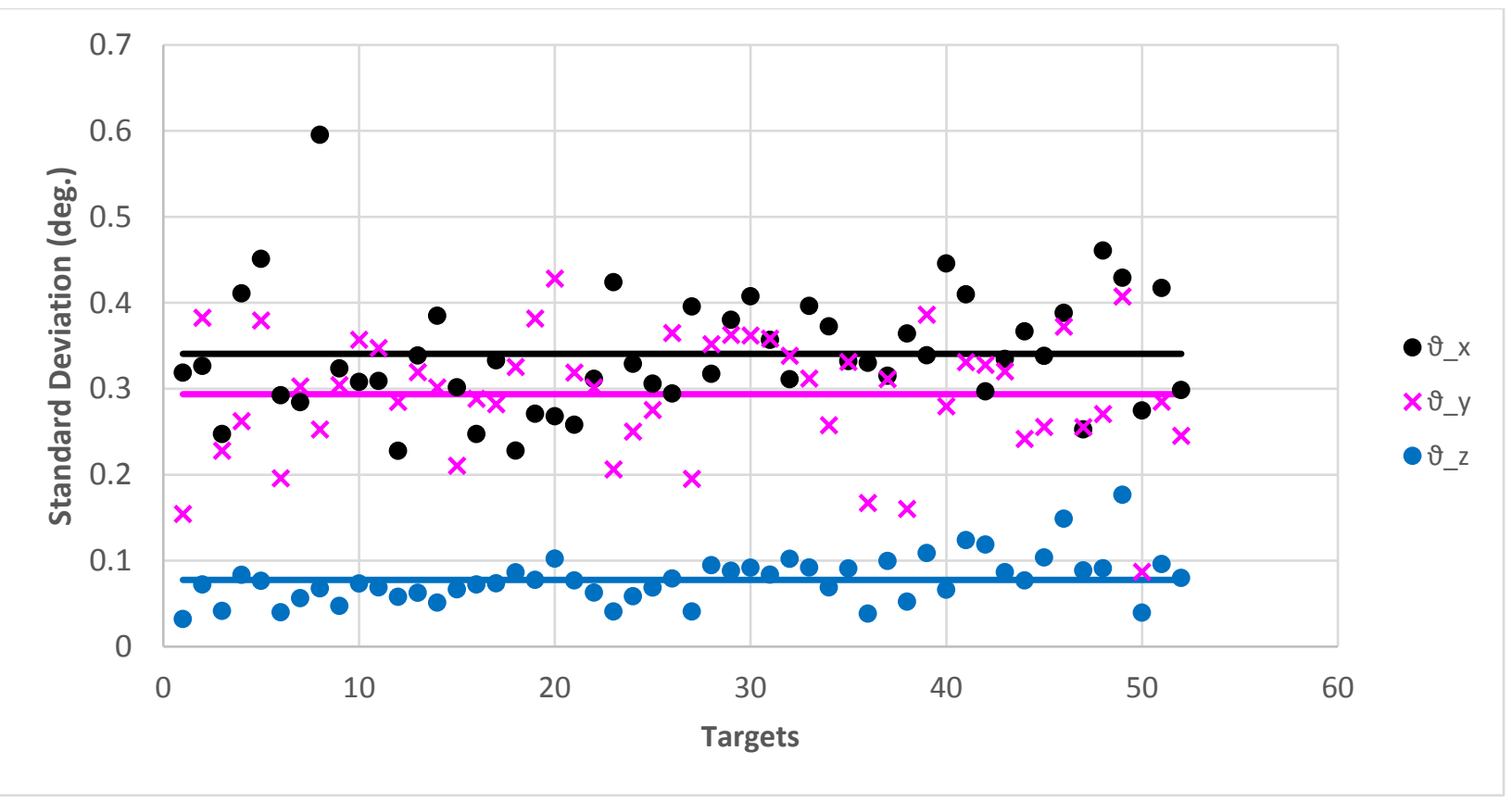

Figure 18. Standard Deviations for the Rotation for 52 targets.

\section{SUMMARY AND FUTURE PLANS}

Three peg-in-hole experiments were conducted to assess the performance of the RRBC method using the performance of the conventional, point-based, rigid body registration method as a baseline. A total of 277 fiducial and 80 target points were measured with a 6 DOF collaborative robot arm in position control mode with the nominal positions on an optical table serving as ground truth positions. A Pass/Fail metric was used to determine the performance of a method. Additionally, two other metrics, $T R E$ and $R M S_{T}$, were also used to assess the performance of the methods.

The peg-in-hole experiments showed that the RRBC method greatly improved the conventional registration method. The method reduced the failed insertions by about $15 \%$ when the Original angles were used and by about $31 \%$ when Fixed angles were used. The use of Fixed angles does not increase the data collection time as the Fixed angles is the average of the Original angles which have to be measured.

The RRBC method has more of an impact when the tolerance on the hole diameter is tighter. This method reduced the percent of failed insertions from $51 \%$ to $13 \%$ for the targets, for Fixed angles. The initial failure rate for tighter tolerances was about $30 \%$ for the conventional 
registration method which may be unacceptable for many applications; thus, necessitating the use of the RRBC method (about $4 \%$ failure).

The RRBC method also reduced the $R M S_{T}$ by about $60 \%$. In most cases, there was a statistically significant positive correlation between TRE and failed positions.

Future plans include repeating the experiments using a 7 DOF collaborative robot arm in impedance control mode, as the use of this type of robot arm is more common in practice for insertion applications. The procedure involves a force-based search pattern to account for misalignment of the peg and the hole, so $100 \%$ successful insertions are expected. In this case, the metrics for comparison between the two methods would be the force required to insert the peg. Also, the use of a robot in impedance control mode would allow for the automated collection of the initial measurements replacing the manually obtained measurements in this report. Subsequent, positions would then be calculated using these measurements and the RRBC method. The automated step would make the process more efficient and therefore more feasible in practical applications.

Other plans also include assessing the RRBC method using a flexible object.

\section{REFERENCES}

1. Van Wyk, K., Culleton, M., Falco, J., and Kelly, K., Comparative Peg-in-Hole Testing of a Force-based Manipulation Controlled Robotic Hand, to be published.

2. De Maeyer, J, Moyaers, B., and Demeester, E, Cartesian Path Planning for Welding Robots: Evaluation of the Descartes Algorithm, IEEE International Conference on Emerging Technologies and Factory Automation, 2017.

3. Levine, S., Pastor, P., Krizhevsky, A., Quillen, D., Learning hand-eye coordination for robotic grasping with deep learning and large-scale data collection, The International Journal of Robotics Research, June, 2017.

4. Ferrari, C., and Canny, J., Planning optimal grasps, IEEE International Conference on Robotics and Automation, 1992.

5. Franaszek, M. and Cheok, G. S., Optimization of Registration Performance Metrics, NISTIR 8111, National Institute of Standards and Technology, Gaithersburg, MD, February, 2016.

6. Franaszek, M., and Cheok, G. S., Method to Improve Point-Based Registration by Restoring Rigid-Body Condition, NISTIR 8180, National Institute of Standards and Technology, Gaithersburg, MD, June, 2017.

7. Fitzpatrick, J. M., West, J. B., and Maurer, C. R., Predicting Error in Rigid-Body PointBased Registration, IEEE Transactions on Medical Imaging, Vol. 17, No. 5, October, 1998. 
8. Van Wyk, K., and Marvel, J. A., Strategies for Improving and Evaluating Robot Registration Performance, IEEE Transactions on Automation Science and Engineering, 2017. 


\section{APPENDIX}

The locations of failed insertions for all runs and for all experiments and the run in which an insertion failed are given in Figures A-2 to A-5. The run in which the insertion failed may be determined from the marker position around a target as described and shown graphically in Figure A-1.

In Figures A-2 to A-5, the target locations are the intersections of labeled vertical lines $(\mathrm{C}-\mathrm{U})$ and numbered horizontal lines (22 - 34). Lines with no numbers or letters do not contain target locations; recall from Figure 2 that targets are located in every other row and column. For example, target locations are C-20, C-22, E-32, and S-28.

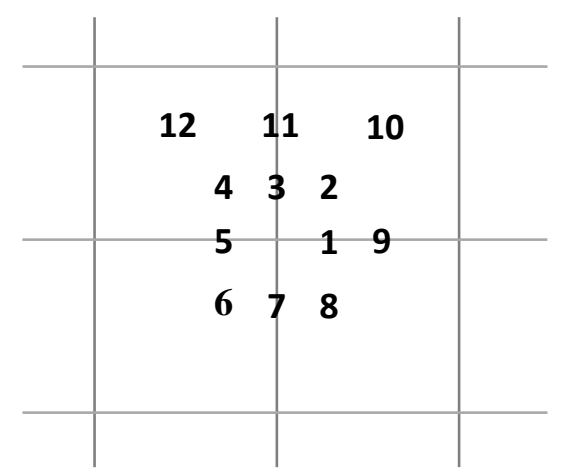

a. At a given target, the location of the marker corresponds to a run number as shown above. Note that Exp. 1 and 2 have 7 runs each and Exp. 3 has 12 runs.

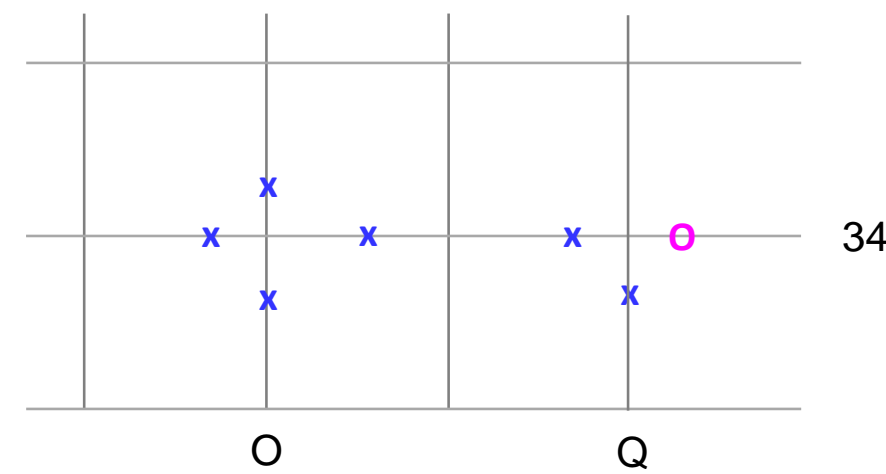

b. Example in Figure A-5 and reproduced here:

1. Target O-34, uncorrected failed insertions occurred in runs $3,5,7$, and 9.

2. Target Q-34 uncorrected failed insertions occurred in runs 5 and 7 and run 1 for corrected.

Figure A-1. Magnified View of a Failed Location Mapping Failed Insertions to Run Numbers. 


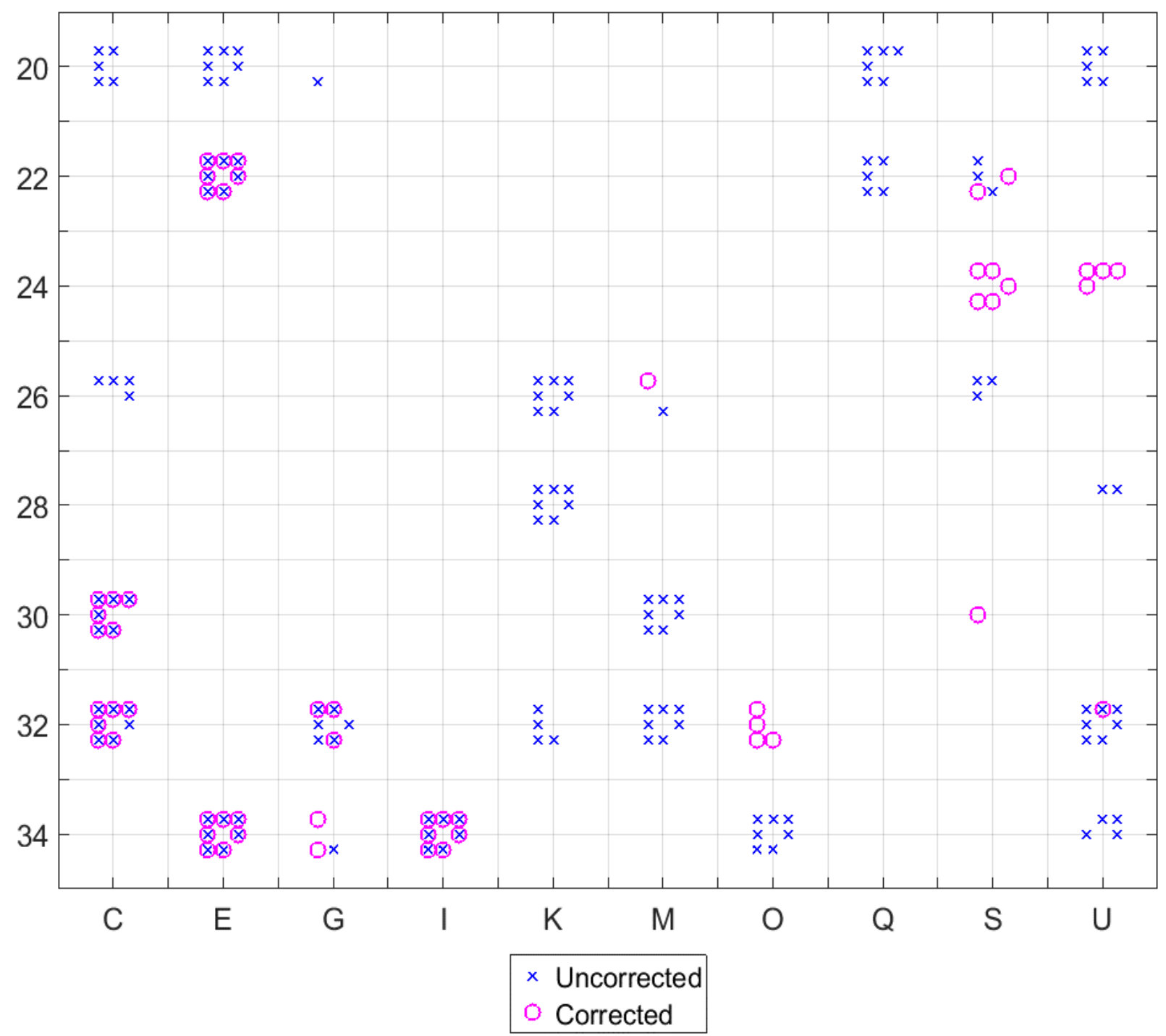

Figure A-2. Failed Locations for Runs 1-7, Experiment 1, Original Angles. 


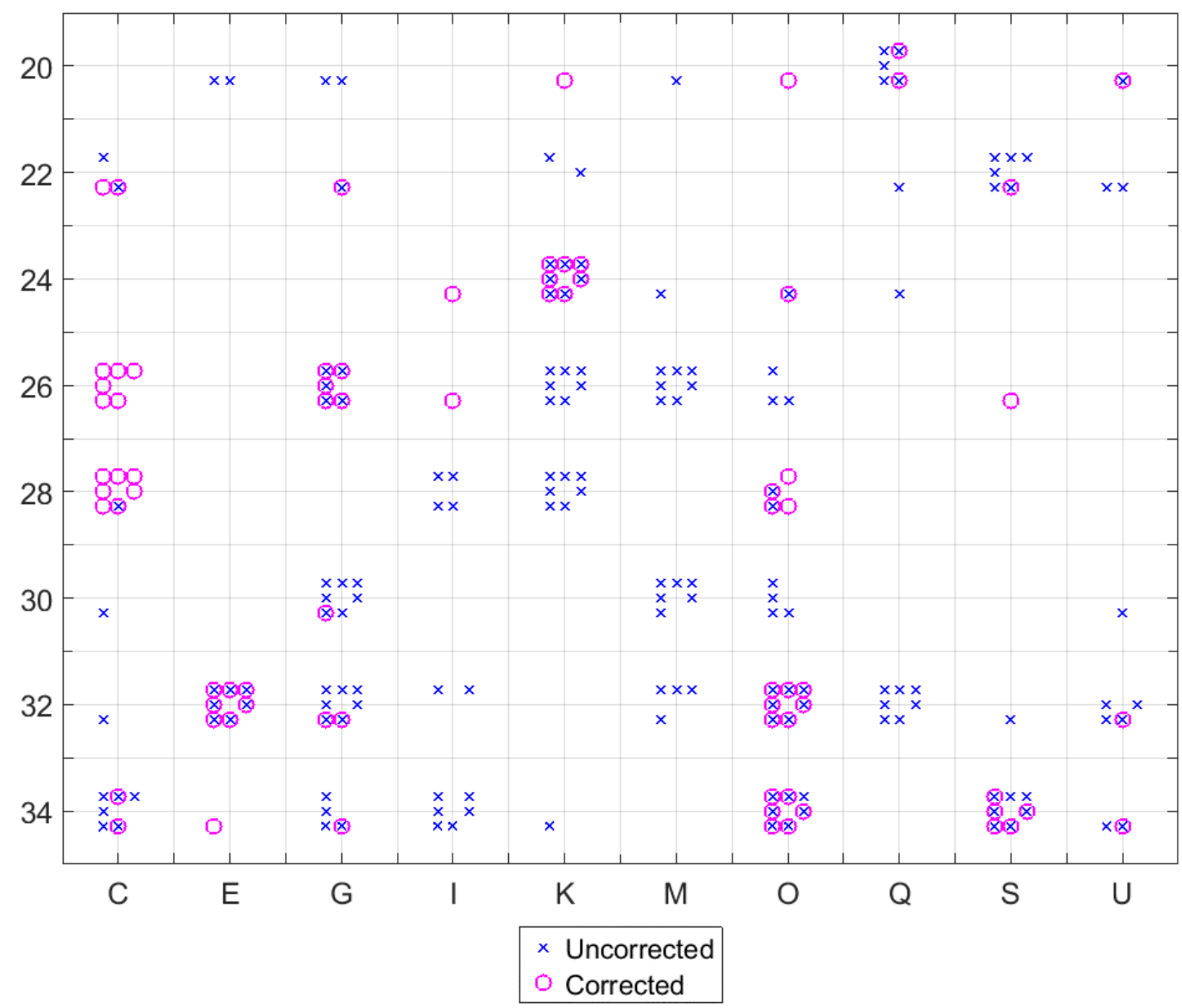

Figure A-3. Failed Locations for Runs 1-7, Experiment 2, Original Angles. 


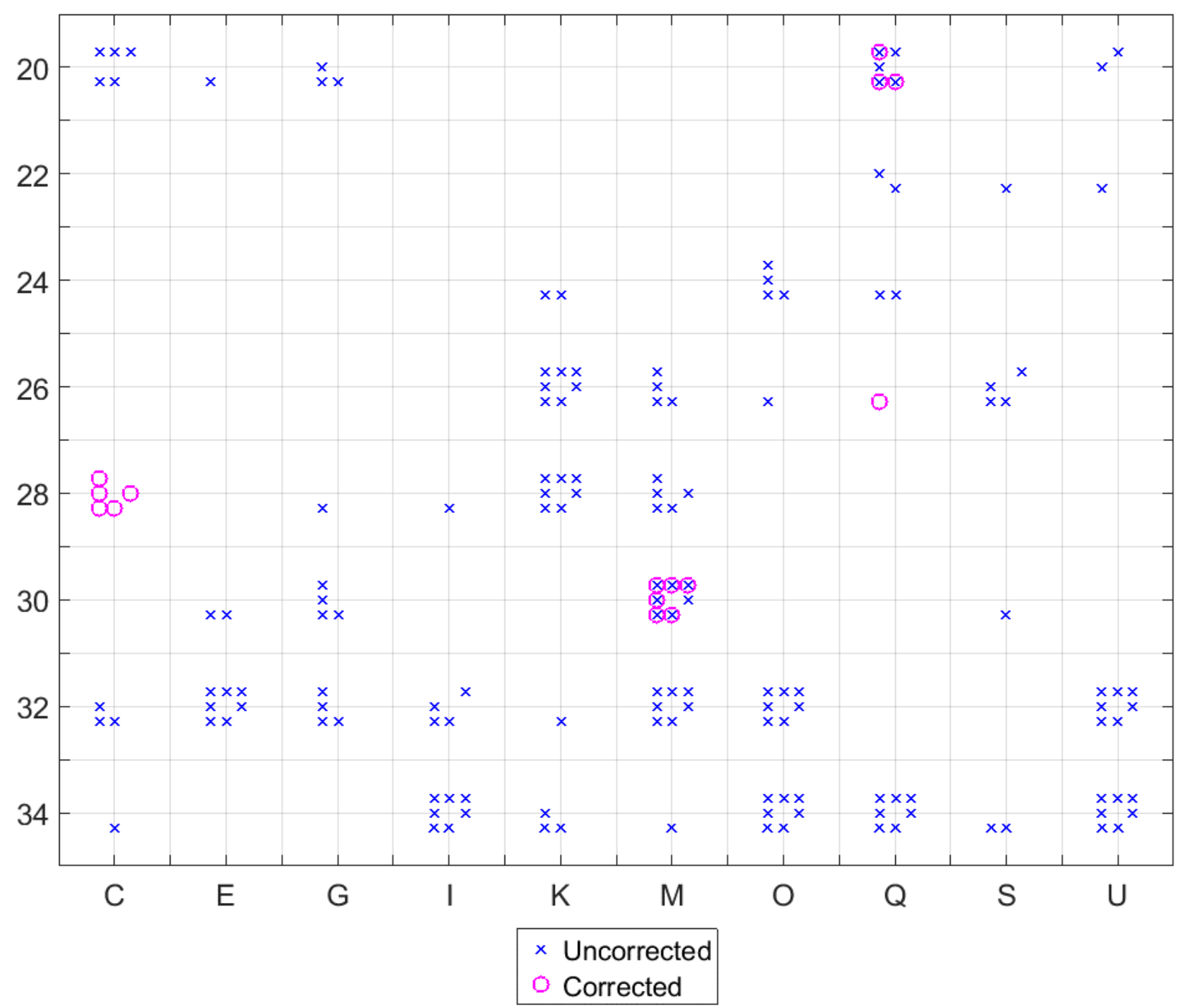

Figure A-4. Failed Locations for Runs 1-7, Experiment 2, Fixed Angles. 


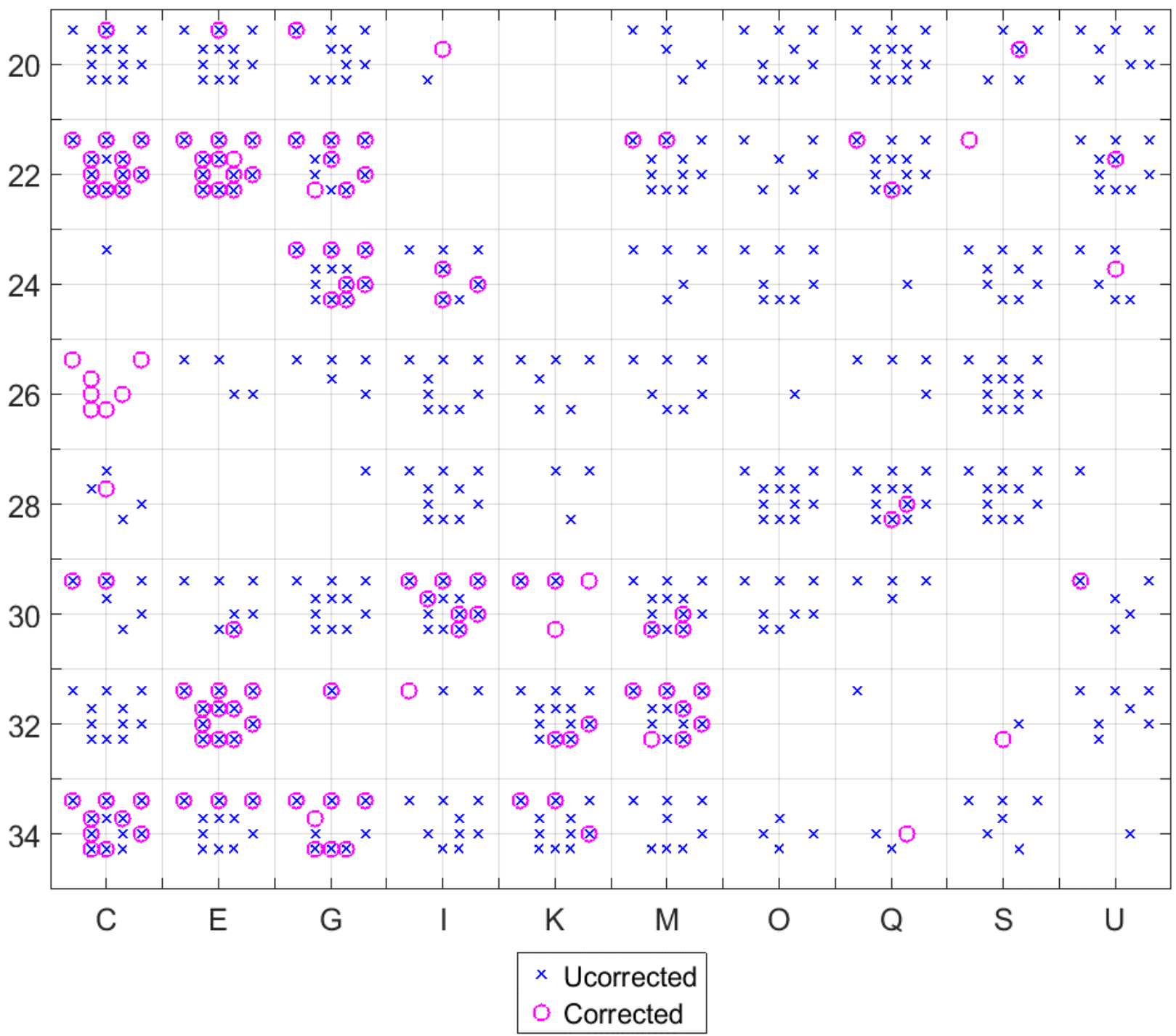

Figure A-5. Failed Locations for Runs 1-12, Experiment 3, Fixed Angles. 